\title{
Semicontinuity of Singularity Invariants in Families of Formal Power Series
}

\author{
Gert-Martin Greuel and Gerhard Pfister \\ Dedicated to András Némethi on the occasion of his sixtieth birthday
}

\begin{abstract}
The problem we are considering came up in connection with the classification of singularities in positive characteristic. Then it is important that certain invariants like the determinacy can be bounded simultaneously in families of formal power series parametrized by some algebraic variety. In contrast to the case of analytic or algebraic families, where such a bound is well known, the problem is rather subtle, since the modules defining the invariants are quasi-finite but not finite over the base space. In fact, in general the fibre dimension is not semicontinuous and the quasi-finite locus is not open. However, if we pass to the completed fibres in a family of rings or modules we can prove that their fibre dimension is semicontinuous under some mild conditions. We prove this in a rather general framework by introducing and using the completed and the Henselian tensor product, the proof being more involved than one might think. Finally we apply this to the Milnor number and the Tjurina number in families of hypersurfaces and complete intersections and to the determinacy in a family of ideals.
\end{abstract}

Mathematics Subject Classification (2000). 13B35, 13B40, 14A15, 14B05, 14 B07.

Keywords. Formal power series, completed tensor product, Henselian tensor product, semicontinuity, Milnor number, Tjurina number, determinacy.

Table of Contents

Introduction

1. Quasi-finite modules and semicontinuity 3

1.1. The completed tensor product 3

$\begin{array}{lll}\text { 1.2. } & \text { Fibre and completed fibre } & 7\end{array}$

1.3. Semicontinuity over a 1-dimensional ring 14

1.4. Henselian rings and Henselian tensor product 19

1.5. Semicontinuity for algebraically presented modules 21 
1.6. Related results 26

2. Singularity invariants 28

2.1. Isolated singularities and flatness 28

2.2. Milnor number and Tjurina number of hypersurface singularities 32

2.3. Determinacy of ideals 33

2.4. Tjurina number of complete intersection singularities 34

References $\quad 36$

\section{Introduction}

In connection with the classification of singularities defined by formal power series over a field a fundamental invariant is the modality of the singularity (with respect to some equivalence relation like right or contact equivalence). To determine the modality one has to investigate adjacent singularities that appear in nearby fibres. This cannot be done by considering families over complete local rings but one has to consider families of power series parametrized by some algebraic variety in the neighbourhood of a given point. To determine potential adjacencies, an important tool is the semicontinuity of certain singularity invariants like, for example, the Milnor or the Tjurina number. Another basic question is if the determinacy of an ideal can be bounded by a semicontinuous invariant. In the complex analytic situation the answer to these questions is well known and positve, but for formal power series the problem is much more subtle than one might think at the first glance. This is mainly due to the fact that ideals or modules that define the invariants are quasi-finite but not finite over the base space.

The modality example shows that the questions treated in this paper are rather natural and appear in important applications. Moreover, the semicontinuity in general is a very basic property with numerous applications in many other contexts. Therefore we decided to choose a rather general framework with families of modules presented by matrices of power series and parametrized by the spectrum of some Noetherian ring. It is not difficult to see that the fibre dimension is in general not semicontinuous and that the quasi-finite locus is in general not open (in contrast the case of ring maps of finite type, where the quasi-finite locus is open by Zariski's Main Theorem, cf. Proposition 47), see Examples 19 and 20. It turns out that the situation is much more satisfactory if we consider not the fibres but the completed fibres and we prove the desired semicontinuity for the completed fibre dimension under some conditions on the family. To guarantee that the completed fibre families behave well under base change we introduce the notion of a (partial) completed tensor product and study its properties in sections 1.1 and 1.2 .

Unfortunately, we cannot prove the semicontinuity of the completed fibre dimension in full generality. We prove it if either the base ring has dimension one (in section 1.3), or if the base ring is complete local containing a field, or if 
the presentation matrix has polynomials or algebraic power series as entries (in section 1.5). Together, these cases cover most applications (see Corollary 45 for a summary). To treat the latter case, we use Henselian rings and the Henselian tensor product, for which we give a short account in section 1.4. It would be interesting to know, if the result holds for presentation matrices with arbitrary power series as entries or if there are counterexamples. In section 1.6 we consider also the case of families of finite type over the base ring and prove a version of Zariski's main theorem for modules. Moreover, we compare the completed fibre with the usual fibre.

In section 2 we apply our results to singularity invariants. We discuss and compare first the notions of regularity and smoothness (over a field) and show that both notions coincide for the completed fibres (Lemma 61). Under the restrictions mentioned above, we prove the semicontinuity of the Milnor number and Tjurina number for hypersurfaces (section 2.2) and the Tjurina number for complete intersections (section 2.4) as well as an upper bound for the determinacy of an ideal (section 2.3). Since the base ring may be the integers, our results are of some interest for computational purposes. For example, if a power series has integer coefficients then the Milnor number over the rationals is bounded by the Milnor number modulo just one (possibly unlucky) prime number if this is finite (see Corollary 64 and, more generally, Corollary 25 and Remark 26).

We assume all rings to be associative, commutative and with unit. Throughout the paper $\mathbb{k}$ denotes an arbitrary field, $A$ a ring, $R=A[[x]], x=\left(x_{1}, \cdots, x_{n}\right)$, the formal power series ring over $A$ and $M$ an $R$-module. For our main results we will assume that $A$ is Noetherian and that $M$ is finitely generated as $R$-module.

Acknowledgment: We would like to thank the anonymous referee for their careful proofreading and in particular for providing answers to two of our original questions (cf. the comment after Proposition 21).

\section{Quasi-finite modules and semicontinuity}

\subsection{The completed tensor product}

Let $A$ be a ring, $R=A[[x]]$ and $M$ an $R$-module. For any prime ideal $\mathfrak{p}$ of $A$ let $k(\mathfrak{p})=A_{\mathfrak{p}} / \mathfrak{p} A_{\mathfrak{p}}$ be the residue field of $\mathfrak{p} . k(\mathfrak{p})=\operatorname{Quot}(A / \mathfrak{p})$ is the quotient field of $A / \mathfrak{p}$ and hence $k(\mathfrak{p})=A / \mathfrak{p}$ if $\mathfrak{p}$ is a maximal ideal. We consider $M$ via the canonical map $A \hookrightarrow R$ as an $A$-module and set

$$
M(\mathfrak{p}):=M_{\mathfrak{p}} \otimes_{A_{\mathfrak{p}}} k(\mathfrak{p})=\left(M \otimes_{A} A_{\mathfrak{p}}\right) \otimes_{A_{\mathfrak{p}}} k(\mathfrak{p})=M \otimes_{A} k(\mathfrak{p}),
$$

which is called the fibre of $M$ over $\mathfrak{p} . M(\mathfrak{p})$ is a vector space over $k(\mathfrak{p})$ and its dimension is denoted by

$$
d_{\mathfrak{p}}(M):=\operatorname{dim}_{k(\mathfrak{p})} M(\mathfrak{p})
$$


$M$ is called quasi-finite ${ }^{1}$ over $\mathfrak{p}$ if $d_{\mathfrak{p}}(M)<\infty$. We are interested in the behavior of $d_{\mathfrak{p}}(M)$ as $\mathfrak{p}$ varies in $\operatorname{Spec} A$, in particular in finding conditions under which $d_{\mathfrak{p}}(M)$ is semicontinuos on $\operatorname{Spec} A$.

We say that a function $d: \operatorname{Spec} A \rightarrow \mathbb{R}, \mathfrak{p} \mapsto d_{\mathfrak{p}}$, is (upper) semicontinuous at $\mathfrak{p}$ if $\mathfrak{p}$ has an open neighbourhood $U \subset \operatorname{Spec} A$ such that $d_{\mathfrak{q}} \leq d_{\mathfrak{p}}$ for all $\mathfrak{q} \in U$. $d$ is semicontinuous on $\operatorname{Spec} A$ if it is semicontinuous at every $\mathfrak{p} \in \operatorname{Spec} A$.

For finitely presented $A$-modules $M$ the semicontinuity of $\mathfrak{p} \mapsto d_{\mathfrak{p}}(M)$ is true and well known (cf. Lemma 1). However, in many applications $M$ is not finitely generated over $A$ but finite over some $A$-algebra $R$. Such a situation appears naturally in algebraic geometry, when one considers families of schemes or of coherent sheaves over Spec $A$. But then it is usually assumed that the $\operatorname{ring} R$ is either of (essentially) finite type over $A$ (in algebraic geometry) or an analytic $A$-algebra (in complex analytic geometry). When we study families of singularities defined by formal power series (cf. Section 2), we have to consider $R=A[[x]]$, which is not of finite type over $A$. As far as we know, this situation has not been systematically studied and it leads to some perhaps unexpected results. For example, $d_{\mathfrak{p}}(M)$ is in general not semicontinuous on Spec $A$ (cf. Examples 19, 20).

It turns out that the situation is much more satisfactory if we pass from the usual fibres to the completed fibres, that is, we consider the completed fibre dimension

$$
\hat{d}_{\mathfrak{p}}(M):=\operatorname{dim}_{k(\mathfrak{p})} M(\mathfrak{p})^{\wedge},
$$

where $M(\mathfrak{p})^{\wedge}$ is the $\langle x\rangle$-adic completion of the $R(\mathfrak{p})$-module $M(\mathfrak{p})$. To guarantee that the completed fibres behave well when $\mathfrak{p}$ varies in $\operatorname{Spec} A$, we introduce the notion of a completed tensor product below.

For a finitely presented $A$-module $M$ the semicontinuity of $\mathfrak{p} \rightarrow d_{\mathfrak{p}}(M)$ is well known:

Lemma 1. If $M$ is a finitely presented A-module then $d_{\mathfrak{p}}(M)$ is semicontinuous on $\operatorname{Spec} A$. Moreover, if $M$ is A-flat, then $d_{\mathfrak{p}}(M)$ is locally constant on $\operatorname{Spec} A$.

Proof. Fix $\mathfrak{p} \in \operatorname{Spec} A$ and consider a presentation of $M$,

$$
A^{p} \stackrel{P}{\rightarrow} A^{q} \rightarrow M \rightarrow 0,
$$

with matrix $P=\left(p_{i j}\right), p_{i j} \in A$. Applying $\otimes_{A} k(\mathfrak{p})$ to this sequence we get the exact sequence of vector spaces

$$
k(\mathfrak{p})^{p} \stackrel{\bar{P}_{\mathfrak{p}}}{\longrightarrow} k(\mathfrak{p})^{q} \rightarrow M(\mathfrak{p}) \rightarrow 0,
$$

with entries of $\bar{P}_{\mathfrak{p}}$ being the images of $p_{i j}$ in $k(\mathfrak{p})$. Then $d_{\mathfrak{p}}(M)$ is finite and $d_{\mathfrak{p}}(M)=q-\operatorname{rank}\left(\bar{P}_{\mathfrak{p}}\right)$. Since $\operatorname{rank}\left(\bar{P}_{\mathfrak{p}}\right) \leq \operatorname{rank}\left(\bar{P}_{\mathfrak{q}}\right)$ for all $\mathfrak{q}$ in some neighbourhood $U$ of $\mathfrak{p}$, the claim follows.

${ }^{1}$ For $M=R / I, I$ an ideal, this is the original definition of Grothendieck. Nowadays most authors (e.g. [Sta19]) require in addition that $R$ is of finite type over $A$. 
If $M$ is flat, then $M_{\mathfrak{p}}$ is free over the local ring $A_{\mathfrak{p}}$ for a given $\mathfrak{p} \in \operatorname{Spec} A$. By [Mat86], Theorem 4.10 (ii) (and its proof) there exists an $f \notin \mathfrak{p}$ such that $M_{f}$ is a free $A_{f}$-module of some rank $r$ and hence $d_{\mathfrak{q}}(M)=r$ for $\mathfrak{q}$ in the open neighbourhood $D(f)$ of $\mathfrak{p}$.

We introduce now the completed tensor product. Let us denote by

$$
\langle x\rangle:=\left\langle x_{1}, \ldots, x_{n}\right\rangle_{R}
$$

the ideal in $R$ generated by $x_{1}, \ldots, x_{n}$. More generally, if $S$ is an $R$-algebra, then $\langle x\rangle_{S}$ denotes the ideal in $S$ generated by (the images of) $x_{1}, \ldots, x_{n}$.

For an $R$-module $N$ denote by

$$
N^{\wedge}:=\lim _{\longleftarrow} N /\langle x\rangle^{m} N
$$

the $\langle x\rangle$-adic completion of $N$. If $N$ is also an $S$-module for some $R$-algebra $S$, then $\langle x\rangle^{m} N=\left(\langle x\rangle_{S}\right)^{m} N$, and hence the $\langle x\rangle$-adic completion and the $\langle x\rangle_{S}$-adic completion of $N$ coincide.

Definition 2. Let $A$ be a ring, $R=A[[x]], B$ an A-algebra and $M$ an $R$-module. We define the completed tensor product of $R$ and $B$ over $A$ as the ring

$$
R \hat{\otimes}_{A} B:=\lim _{\longleftarrow}\left(\left(R /\langle x\rangle^{m}\right) \otimes_{A} B\right)
$$

and the completed tensor product of $M$ and $B$ over $A$ as the module

$$
M \hat{\otimes}_{A} B:=\lim _{\longleftarrow}\left(\left(M /\langle x\rangle^{m} M\right) \otimes_{A} B\right) .
$$

If $N$ is an $A$-module, we define the $R$-module

$$
M \hat{\otimes}_{A} N:=\lim _{\longleftarrow}\left(\left(M /\langle x\rangle^{m} M\right) \otimes_{A} N\right)
$$

and call it the completed tensor product of $M$ and $N$ over $A$.

One reason why we consider the completed tensor product is that it provides the right base change property in the category of rings of the form $A[[x]]$ by the following Proposition 3.1.

Proposition 3. The completed tensor product has the following properties (assumptions as in Definiton 2).

1. $A[[x]] \hat{\otimes}_{A} B=\left(R \otimes_{A} B\right)^{\wedge}=B[[x]]$.

2. $M \hat{\otimes}_{A} N=\left(M \otimes_{A} N\right)^{\wedge}$.

3. If $M$ is finitely presented over $R$ and $N$ is a finitely presented $B$-module, then

$$
M \hat{\otimes}_{A} N \cong\left(M \otimes_{A} N\right) \otimes_{R \otimes_{A} B}\left(R \hat{\otimes}_{A} B\right) .
$$

4. The canonical map $M \otimes_{A} N \rightarrow M \hat{\otimes}_{A} N$ is injective if $A$ is Noetherian, $M$ finite over $R$ and $N$ finite over $A$.

5. If $\langle x\rangle^{m} \subset \operatorname{Ann}_{R}(M)$ for some $m$ then $M \hat{\otimes}_{A} N=M \otimes_{A} N$ for every A-module $N$. 
Proof. 1. We have $\lim \left(A[[x]] /\langle x\rangle^{m} \otimes_{A} B\right)=\lim \left(A[x] /\langle x\rangle^{m} \otimes_{A} B\right)=\lim B[x] /\langle x\rangle^{m}$ $=B[[x]]$, showing the second equality. The first follows since $\left(R /\langle x\rangle^{m}\right) \otimes_{A} B=$ $\left(R \otimes_{A} B\right) /\langle x\rangle^{m}\left(R \otimes_{A} B\right)$.

2. Since $\left(M /\langle x\rangle^{m} M\right) \otimes_{A} N=\left(M \otimes_{A} N\right) /\langle x\rangle^{m}\left(M \otimes_{A} N\right)$ the equality follows

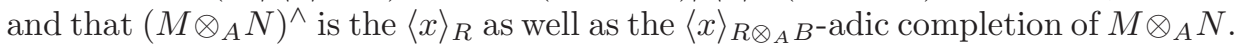

3. If $M$ resp. $N$ are finitely presented over $R$ resp. $B$, then $M \otimes_{A} N$ is finitely presented over $R \otimes_{A} B$. Hence we can apply (the proof of) [AM69, Proposition 10.13] and use 1. to show the isomorphism.

4. If $A$ is Noetherian then $R$ is Noetherian. If $M$ is finitely generated over $R$ and $N$ finitely generated over $A$ then $M \otimes_{A} N$ is finitely generated over $R$. The injectivity follows from 2. and [AM69, Theorem 10.17 and Corollary 10.19], since $\langle x\rangle$ is contained in the Jacobson radical of $R$ by Lemma 16.

5. If $\langle x\rangle^{m} M=0$ for some $m$, then $M \hat{\otimes}_{A} N=M \otimes_{A} N$ by definition of the completed tensor product.

Corollary 4. The completed tensor product is right-exact on the category of finitely presented modules. That is, let

$$
\begin{aligned}
& M^{\prime} \rightarrow M \rightarrow M^{\prime \prime} \rightarrow 0, \text { resp } . \\
& N^{\prime} \rightarrow N \rightarrow N^{\prime \prime} \rightarrow 0
\end{aligned}
$$

be exact sequences of finitely presented $R$-modules resp. B-modules. Then the sequences of finitely presented $R \hat{\otimes}_{A} B$-modules

$$
\begin{aligned}
& M^{\prime} \hat{\otimes}_{A} N \rightarrow M \hat{\otimes}_{A} N \rightarrow M^{\prime \prime} \hat{\otimes}_{A} N \rightarrow 0, \text { resp } . \\
& M \hat{\otimes}_{A} N^{\prime} \rightarrow M \hat{\otimes}_{A} N \rightarrow M \hat{\otimes}_{A} N^{\prime \prime} \rightarrow 0
\end{aligned}
$$

are exact.

Proof. The sequences $M^{\prime} \otimes_{A} N \rightarrow M \otimes_{A} N \rightarrow M^{\prime \prime} \otimes_{A} N \rightarrow 0$ and $M \otimes_{A} N^{\prime} \rightarrow$ $M \otimes_{A} N \rightarrow M \otimes_{A} N^{\prime \prime} \rightarrow 0$ are exact. Now tensor these sequences with $R \hat{\otimes}_{A} B$ over $R \otimes_{A} B$ and apply Proposition 3.3.

\section{Corollary 5.}

(i) $R \hat{\otimes}_{A} A=R$.

(ii) If $S$ is multiplicatively closed in $A$ then $A[[x]] \hat{\otimes}_{A}\left(S^{-1} A\right)=\left(S^{-1} A\right)[[x]]$.

(iii) For any $R$-module $M$ we have $M \hat{\otimes}_{A} A=M^{\wedge}$.

(iv) If $M$ is finitely presented over $R$ then $M=M^{\wedge}$. If moreover $N$ is finitely presented over $A$, then $M \hat{\otimes}_{A} N=M \otimes_{A} N$.

Proof. (i) and (ii) follow immediately from Proposition 3.1, (iii) is a special case of Proposition 3.2 and (iv) follows from (i) and Proposition 3.3 with $B=A$.

Applying Corollary 4 and Proposition 3.1 we get

Corollary 6. If $A[[x]]^{p} \stackrel{T}{\rightarrow} A[[x]]^{q} \rightarrow M \rightarrow 0$ is an $A[[x]]$-presentation of $M$ and $B$ an A-algebra, then

$$
M \hat{\otimes}_{A} B=\operatorname{coker}\left(B[[x]]^{p} \stackrel{T}{\rightarrow} B[[x]]^{q}\right) .
$$


Remark 7. Let $A$ be Noetherian and $B$ an $A$-algebra. If $\langle x\rangle$ is contained in the Jacobson radical of $R \otimes_{A} B$, then $R \hat{\otimes}_{A} B$ is faithfully flat over $R \otimes_{A} B$, by Proposition 3.1 and [Mat86, Theorem 8.14]. Note however that although $\langle x\rangle$ is contained in the Jacobson radical of $R$ by Lemma 16 below, it need not be in the Jacobson radical of $R \otimes_{A} B$ (cf. Example 14).

Example 8. Let $\left\langle f_{1}, \ldots, f_{k}\right\rangle \subset R=A[[x]]$ be an ideal and $M=A[[x]] /\left\langle f_{1}, \ldots, f_{k}\right\rangle$. If $\mathfrak{p}$ is a prime ideal in $A$ then $R \hat{\otimes}_{A} A_{\mathfrak{p}}=A_{\mathfrak{p}}[[x]]$ and form Corollary 6 we get $M \hat{\otimes}_{A} A_{\mathfrak{p}}=A_{\mathfrak{p}}[[x]] /\left\langle f_{1}, \ldots, f_{k}\right\rangle$. If $k(\mathfrak{p})$ is the residue field of $A$ at $\mathfrak{p}$ then $M \hat{\otimes}_{A} k(\mathfrak{p})=k(\mathfrak{p})[[x]] /\left\langle f_{1}, \ldots, f_{k}\right\rangle$, something what one expects as fibre of $M$ over $\mathfrak{p}$. While $A_{\mathfrak{p}}[[x]]$ and $k(\mathfrak{p})[[x]]$ are nice local rings, the subrings $R \otimes_{A} A_{\mathfrak{p}} \subset A_{\mathfrak{p}}[[x]]$ and $R \otimes_{A} k(\mathfrak{p}) \subset k(\mathfrak{p})[[x]]$ are in general not local if $\mathfrak{p}$ is not a maximal ideal (see Example 14).

Remark 9. Proposition 3.1 with $B=A[[y]], y=\left(y_{1}, \ldots, y_{m}\right)$, implies

$$
A[[x]] \hat{\otimes}_{A} A[[y]]=A[[x, y]] .
$$

Now let $A$ be Noetherian. If $I$ resp. $J$ are ideals in $A[[x]]$ resp. $A[[y]]$, we get from Corollary 4

$$
A[[x]] / I \hat{\otimes}_{A} A[[y]] / J=A[[x, y]] /\langle I, J\rangle A[[x, y]] .
$$

We call an $A$-algebra a formal $A$-algebra if it is isomorphic to an $A$-algebra $A[[x]] / I$. For two formal $A$-algebras $B=A[[x]] / I$ and $C=A[[y]] / J$ the completed tensor product can be defined as $B \hat{\otimes}_{A} C=A[[x, y]] /\langle I, J\rangle A[[x, y]]$. It has the usual universal property of the tensor product in the category of formal $A$-algebras, analogous to the analytic tensor product for analytic algebras (cf. [GR71, Chapter III.5]). Thus, Definition 2 generalizes the completed tensor product of formal $A$-algebras.

\subsection{Fibre and completed fibre}

Let again $A$ be a ring and $M$ an $R=A[[x]]$-module. We introduce the completed fibre $\hat{M}(\mathfrak{p})$ and the completed fibre dimension $\hat{d}_{\mathfrak{p}} M$ of $M$ for $\mathfrak{p} \in \operatorname{Spec} A$ and compare it with the usual fibre $M(\mathfrak{p})$ and the usual fibre dimension $d_{\mathfrak{p}} M$.

At the end of this section we give examples, showing that semicontinuity of $d_{\mathfrak{p}}(M)$ does not hold in general on $\operatorname{Spec} A$, even if $A=\mathbb{C}[t]$ or $A=\mathbb{Z}$ (Examples 19 and 20). However, we show in the next sections 1.3 and 1.5 that, under some conditions, semicontinuity holds for the completed fibre dimension $\hat{d}_{\mathfrak{p}}(M)$.

Notation 10. We have canonical maps

$$
A \stackrel{j}{\hookrightarrow} R \stackrel{\pi}{\longrightarrow} R /\langle x\rangle \stackrel{i}{\cong} A,
$$

with $i \circ \pi \circ j=i d$ and for an ideal $I \subset R$ we set $\bar{I}:=\pi(I)$. On the level of schemes we have the maps $\operatorname{Spec} A \stackrel{i^{*}}{\cong} V(\langle x\rangle) \stackrel{\pi^{*}}{\longrightarrow} \operatorname{Spec} R \stackrel{j^{*}}{\longrightarrow} \operatorname{Spec} A$, with $i^{*}(\mathfrak{p})=$ $\langle\mathfrak{p}, x\rangle, \quad j^{*}(\langle\mathfrak{p}, x\rangle)=\langle\mathfrak{p}, x\rangle \cap A=\mathfrak{p}$ for $\mathfrak{p} \in \operatorname{Spec} A$. We denote by

$$
\mathfrak{n}_{\mathfrak{p}}:=\langle\mathfrak{p}, x\rangle=\left\langle\mathfrak{p}, x_{1}, \ldots, x_{n}\right\rangle_{R}
$$


the ideal in $R$ generated by $\mathfrak{p} \in \operatorname{Spec} A$ and $x_{1}, \ldots, x_{n}$. The family

$$
f:=j^{*}: \operatorname{Spec} R \rightarrow \operatorname{Spec} A
$$

has the trivial section $\sigma=(i \circ \pi)^{*}: \operatorname{Spec} A \rightarrow \operatorname{Spec} R, \mathfrak{p} \mapsto \mathfrak{n}_{\mathfrak{p}}$, and the composition $h:=\pi \circ j: A \cong R /\langle x\rangle$ induces an isomorphism

$$
h^{*}: V(\langle x\rangle) \stackrel{\cong}{\longrightarrow} \operatorname{Spec} A,
$$

the restriction of $f$ to $V(\langle x\rangle)$. We call $R_{\mathfrak{p}}:=R \otimes_{A} A_{\mathfrak{p}}$ the stalk of $R$ over $\mathfrak{p}$. $R_{\mathfrak{p}}$ is not a local ring, its local ring at $\mathfrak{n}_{\mathfrak{p}}$ is $\left(R_{\mathfrak{p}}\right)_{\mathfrak{n}_{\mathfrak{p}}}=R_{\mathfrak{n}_{\mathfrak{p}}}$ with residue field $k\left(\mathfrak{n}_{\mathfrak{p}}\right)=k(\mathfrak{p})$ (by Lemma 12 below).

If $M$ is an $R$-module, we call $M_{\mathfrak{p}}=M \otimes_{A} A_{\mathfrak{p}}$ the stalk of $M$ over $\mathfrak{p}$ and we are interested in the behavior of $M$ along the section $\sigma$. However, we are not interested in the $R(\mathfrak{p})$-modules $M(\mathfrak{p})$ since $R(\mathfrak{p})$ is not a power series ring (and does not behave nicely). We are interested in the completed stalk $\hat{M}_{\mathfrak{p}}$ and in the completed fibres $\hat{M}(\mathfrak{p})$, which we introduce now.

Definition 11. Let $A$ be a ring, $R=A[[x]], M$ an $R$-module and $\mathfrak{p} \in \operatorname{Spec} A$.

1. We set $\hat{R}_{\mathfrak{p}}:=R \hat{\otimes}_{A} A_{\mathfrak{p}}$, a local ring isomorphic to $A_{\mathfrak{p}}[[x]]$ (Proposition 3.1), and call the $\hat{R}_{\mathfrak{p}}$-module

$$
\hat{M}_{\mathfrak{p}}:=M \hat{\otimes}_{A} A_{\mathfrak{p}}
$$

the completed stalk of $M$ over $\mathfrak{p}$.

2. The ring $\hat{R}(\mathfrak{p}):=R \hat{\otimes}_{A} k(\mathfrak{p})$ is called the completed fibre of $R$ over $\mathfrak{p}$. It is a local ring isomorphic to $k(\mathfrak{p})[[x]]$ (Proposition 3.1). The $\hat{R}(\mathfrak{p})$-module

$$
\hat{M}(\mathfrak{p}):=M \hat{\otimes}_{A} k(\mathfrak{p})=\hat{M}_{\mathfrak{p}} \otimes_{A_{\mathfrak{p}}} k(\mathfrak{p})
$$

is called the completed fibre of $M$ over $\mathfrak{p}$.

3. $\hat{M}(\mathfrak{p})$ is a $k(\mathfrak{p})$-vector space and we call its dimension

$$
\hat{d}_{\mathfrak{p}}(M):=\operatorname{dim}_{k(\mathfrak{p})} \hat{M}(\mathfrak{p})
$$

the completed fibre dimension of $M$ over $\mathfrak{p}$.

4. $M$ is called quasi-completed-finite over $\mathfrak{p}$ if $\hat{d}_{\mathfrak{p}}(M)<\infty$.

The map $A \rightarrow R$ induces a map of local rings $A_{\mathfrak{p}} \rightarrow R_{\mathfrak{n}_{\mathfrak{p}}}$ and for an $R$-module $M$ we have the fibre $M(\mathfrak{p})=M \otimes_{A} k(\mathfrak{p})$ of $M$ w.r.t. $A \rightarrow R$ and the fibre

$$
M_{\mathfrak{n}_{\mathfrak{p}}}(\mathfrak{p})=M_{\mathfrak{n}_{\mathfrak{p}}} \otimes_{A_{\mathfrak{p}}} k(\mathfrak{p})=M_{\mathfrak{n}_{\mathfrak{p}}} / \mathfrak{p} M_{\mathfrak{n}_{\mathfrak{p}}}
$$

of $M_{\mathfrak{n}_{\mathfrak{p}}}$ w.r.t. $A_{\mathfrak{p}} \rightarrow R_{\mathfrak{n}_{\mathfrak{p}}}$. The fibres are in general different but the completed fibres coincide by Lemma 15 if $M$ is finitely $R$-presented.

Let us first compare the fibre $M(\mathfrak{p})$ with its completed fibre $\hat{M}(\mathfrak{p})$.

Lemma 12. For any $R$-module $M$ the following holds.

(i) $\hat{M}_{\mathfrak{p}}=\left(M_{\mathfrak{p}}\right)^{\wedge}$ and $\hat{M}(\mathfrak{p})=M(\mathfrak{p})^{\wedge}$.

(ii) $\mathfrak{n}_{\mathfrak{p}}$ is a prime ideal in $R$ with $\mathfrak{n}_{\mathfrak{p}} \cap A=\mathfrak{p}$ and the residue field of $\mathfrak{n}_{\mathfrak{p}}$ in $R$ satisfies $k\left(\mathfrak{n}_{\mathfrak{p}}\right)=k(\mathfrak{p})$. 
(iii) If $\mathfrak{n}$ is any prime ideal in $R$ containing $\langle x\rangle$, then $\mathfrak{n}=\mathfrak{n}_{\mathfrak{p}}$ with $\mathfrak{p}=\mathfrak{n} \cap A \in$ $\operatorname{Spec} A$.

Proof. Statement (i) follows from Proposition 3.1. The first statement of (ii) follows since $R / \mathfrak{n}_{\mathfrak{p}}=A / \mathfrak{p}$ is an integral domain. Since $R / \mathfrak{n}_{\mathfrak{p}}=A / \mathfrak{p}$ we have $k\left(\mathfrak{n}_{\mathfrak{p}}\right)=$ $\operatorname{Quot}\left(R / \mathfrak{n}_{\mathfrak{p}}\right)=\operatorname{Quot}(A / \mathfrak{p})=k(\mathfrak{p})$. (iii) is obvious.

Remark 13. We have strict flat inclusions $A_{\mathfrak{p}} \varsubsetneqq R_{\mathfrak{p}} \varsubsetneqq R_{\mathfrak{n}_{\mathfrak{p}}} \varsubsetneqq A_{\mathfrak{p}}[[x]]$ of rings that are Noetherian if $A$ is Noetherian.

The strictness is easy to see. E.g. $g_{0}+\sum_{|\alpha|>1}\left(g_{\alpha} / h_{\alpha}\right) x^{\alpha}, g_{0} \notin \mathfrak{p}$, with arbitrary $h_{\alpha} \in R \backslash \mathfrak{n}_{\mathfrak{p}}$, is a unit in $A_{\mathfrak{p}}[[x]]$ but it is not contained in $R_{\mathfrak{n}_{\mathfrak{p}}}$, where only finitely many different denominators are allowed. We have $R_{\mathfrak{p}}=S^{-1} R$, with $S$ the multiplictive set $A \backslash \mathfrak{p}$ and $R_{\mathfrak{n}_{\mathfrak{p}}}=\left(R_{\mathfrak{p}}\right)_{\mathfrak{n}_{\mathfrak{p}}}$. Since localization preserves flatness ([AM69, Corollary 3.6]) and the Noether property ([AM69, Proposition 7.3]), the inclusions $A_{\mathfrak{p}} \subset R_{\mathfrak{p}} \subset R_{\mathfrak{n}_{\mathfrak{p}}}$ are flat and the rings are Noetherain if $A$ is Noetherain. The flatness of $A_{\mathfrak{p}}[[x]]$ over $R_{\mathfrak{n}_{\mathfrak{p}}}$ follows, since the first is the $\langle x\rangle$-adic completion of the second by Lemma 12 (i). Since both rings are local, $R_{\mathfrak{n}_{\mathfrak{p}}} \subset A_{\mathfrak{p}}[[x]]$ is faithfully flat.

The rings $R_{\mathfrak{p}}$ and $R_{\mathfrak{n}_{\mathfrak{p}}}$ are "strange" subrings of $A_{\mathfrak{p}}[[x]]$. The ring $A_{\mathfrak{p}}[[x]]$ is of interest in applications (cf. section 2), while the rings $R_{\mathfrak{p}}$ and $R_{\mathfrak{n}_{\mathfrak{p}}}$ are of minor interest. By the following Lemma 15 we have $\left(R_{\mathfrak{p}}\right)^{\wedge}=\left(R_{\mathfrak{n}_{\mathfrak{p}}}\right)^{\wedge}=A_{\mathfrak{p}}[[x]]$.

Example 14. As an example let $A=\mathbb{k}[t]$ and $R=A[[x]]$ with $t$ and $x$ one variable, $\mathfrak{p}=\langle 0\rangle \in \operatorname{Spec} A$. We have $A_{\mathfrak{p}}=k(\mathfrak{p})=\mathbb{k}(t)$ and

$$
R_{\mathfrak{p}}=\mathbb{k}[t][[x]] \otimes_{\mathbb{k}[t]} \mathbb{k}(t)=\{g / h \mid g \in \mathbb{k}[t][[x]], h \in \mathbb{k}[t] \backslash 0\},
$$

$g=g_{0}+\sum_{i \geq 1} g_{i} x^{i}, g_{i} \in \mathbb{k}[t]$, a subring strictly contained in $R \hat{\otimes}_{A} A_{\mathfrak{p}}=\mathbb{k}(t)[[x]]$.

- $\langle x\rangle$ is contained in the Jacobson radical of $R \hat{\otimes}_{A} A_{\mathfrak{p}}$ by Lemma 16.

- $\langle x\rangle$ is not contained in the Jacobson radical of $R_{\mathfrak{p}}=R \otimes A_{\mathfrak{p}}$.

To see this, note that the element $t-x$ is a unit in $R \hat{\otimes}_{A} A_{\mathfrak{p}}$, since $1 /(t-x)=$ $1 / t \sum_{i>0}(x / t)^{i}$, but $1 /(t-x)$ is not an element in $R_{\mathfrak{p}}$. The ideal $\langle t-x\rangle$ is a maximal ideal in $R_{\mathfrak{p}}$, since $R_{\mathfrak{p}} /\langle t-x\rangle \cong \mathbb{k}_{k}((t))$ (see Example 19.2), but $x \notin \mathfrak{m}$ since otherwise $t \in \mathfrak{m}$, contradicting the fact that $t$ is a unit $R_{\mathfrak{p}}$.

- The rings $R_{\mathfrak{p}}$ and $R(\mathfrak{p})$ are in general not local.

Since $R_{\mathfrak{p}} /\langle x\rangle=\mathbb{k}(t)$, the ideals $\langle x\rangle$ and $\langle t-x\rangle$ are two different maximal ideals and $R_{\mathfrak{p}}=R(\mathfrak{p})(\mathfrak{p}=\langle 0\rangle)$ is not local.

Lemma 15. Let $M$ be a finitely presented $R$-module and $\mathfrak{p} \in \operatorname{Spec} A$.

1. We have isomorphisms

$$
\hat{M}_{\mathfrak{p}} \cong M_{\mathfrak{n}_{\mathfrak{p}}} \hat{\otimes}_{A_{\mathfrak{p}}} A_{\mathfrak{p}}=M_{\mathfrak{n}_{\mathfrak{p}}} \hat{\otimes}_{A} A=\left(M_{\mathfrak{n}_{\mathfrak{p}}}\right)^{\wedge} .
$$

2. $\hat{M}(\mathfrak{p}) \cong\left(M_{\mathfrak{n}_{\mathfrak{p}}} / \mathfrak{p} M_{\mathfrak{n}_{\mathfrak{p}}}\right)^{\wedge}$. 
3. If $M=\operatorname{coker}\left(A[[x]]^{p} \stackrel{T}{\rightarrow} A[[x]]^{q}\right)$ then

$$
\begin{gathered}
\hat{M}_{\mathfrak{p}}=M \hat{\otimes}_{A} A_{\mathfrak{p}}=\operatorname{coker}\left(A_{\mathfrak{p}}[[x]]^{p} \stackrel{T}{\rightarrow} A_{\mathfrak{p}}[[x]]^{q}\right), \\
\hat{M}(\mathfrak{p})=\operatorname{coker}\left(k(\mathfrak{p})[[x]]^{p} \stackrel{T}{\rightarrow} k(\mathfrak{p})[[x]]^{q}\right) .
\end{gathered}
$$

Note that $\hat{R}_{\mathfrak{p}}=A_{\mathfrak{p}}[[x]]=\left(R_{\mathfrak{p}}\right)^{\wedge} \cong\left(R_{\mathfrak{n}_{\mathfrak{p}}}\right)^{\wedge}$ and $\hat{R}(\mathfrak{p})=k(\mathfrak{p})[[x]]=R(\mathfrak{p})^{\wedge} \cong$ $\left(R_{\mathfrak{n}_{\mathfrak{p}}} / \mathfrak{p} R_{\mathfrak{n}_{\mathfrak{p}}}\right)^{\wedge}$ are local rings but $R_{\mathfrak{p}} \neq R_{\mathfrak{n}_{\mathfrak{p}}}$ and $R(\mathfrak{p}) \neq R_{\mathfrak{n}_{\mathfrak{p}}} / \mathfrak{p} R_{\mathfrak{n}_{\mathfrak{p}}}$, since $R_{\mathfrak{p}}$ and $R(\mathfrak{p})$ are in general not local.

Proof. 1. The natural inclusion $R_{\mathfrak{n}_{\mathfrak{p}}}=A[[x]]_{\mathfrak{n}_{\mathfrak{p}}} \hookrightarrow A_{\mathfrak{p}}[[x]]$ is given as follows. Let $h / g \in R_{\mathfrak{n}_{\mathfrak{p}}}$ with $h, g \in R, g \notin \mathfrak{n}_{\mathfrak{p}}$ and write $g=g_{0}-g_{1}$ with $g_{0} \in A$ and $g_{1} \in\langle x\rangle R$. Then $g \notin \mathfrak{n}_{\mathfrak{p}}=\langle\mathfrak{p}, x\rangle$ iff $g_{0} \notin \mathfrak{p}$ and $g$ is a unit in $R_{\mathfrak{n}_{\mathfrak{p}}}$ iff its image in $A_{\mathfrak{p}}[[x]]$ is a unit. We get

$$
h / g=\frac{g_{0}^{-1} h}{\left(1-g_{1} / g_{0}\right)}=g_{0}^{-1} h \sum_{i \geq 0}\left(g_{1} / g_{0}\right)^{i} \in A_{\mathfrak{p}}[[x]] .
$$

Now it is not difficult to see that the induced map $A[[x]]_{\mathfrak{n}_{\mathfrak{p}}} /\langle x\rangle^{m} \rightarrow A_{\mathfrak{p}}[[x]] /\langle x\rangle^{m}$ is bijective (a finite sum $\sum_{|\alpha|=0}^{m-1}\left(a_{\alpha} / b_{\alpha}\right) x^{\alpha}, a_{\alpha}, b_{\alpha} \in A, b_{\alpha} \notin \mathfrak{p}$ in $A_{\mathfrak{p}}[[x]]$ can be written as $1 / b \sum_{|\alpha|=0}^{m-1}\left(a_{\alpha} b_{\alpha}^{\prime}\right) x^{\alpha}$ with $b=\prod b_{\alpha} \notin \mathfrak{n}_{\mathfrak{p}}, b_{\alpha}^{\prime}=b / b_{\alpha} \in A$, and hence is in $\left.A[[x]]_{\mathfrak{n}_{\mathfrak{p}}}\right)$. We get

$$
R_{\mathfrak{n}_{\mathfrak{p}}} \hat{\otimes}_{A} A=\lim _{\longleftarrow} A[[x]]_{\mathfrak{n}_{\mathfrak{p}}} /\langle x\rangle^{m} \otimes_{A} A=\lim _{\longleftarrow} A_{\mathfrak{p}}[[x]] /\langle x\rangle^{m}=A_{\mathfrak{p}}[[x]]
$$

and also $R_{\mathfrak{n}_{\mathfrak{p}}} \hat{\otimes}_{A_{\mathfrak{p}}} A_{\mathfrak{p}}=A_{\mathfrak{p}}[[x]]=R_{\mathfrak{n}_{\mathfrak{p}}}^{\wedge}$. Now apply Corollary 4 to the presentation of $M$ and deduce the claim for $M \hat{\otimes}_{A} A_{\mathfrak{p}}$.

2. $\hat{M}(\mathfrak{p})=M \hat{\otimes}_{A}\left(A_{\mathfrak{p}} / \mathfrak{p} A_{\mathfrak{p}}\right)=\left(M \hat{\otimes}_{A} A_{\mathfrak{p}}\right) / \mathfrak{p}\left(M \hat{\otimes}_{A} A_{\mathfrak{p}}\right)=M_{\mathfrak{n}_{\mathfrak{p}}}^{\wedge} / \mathfrak{p} M_{\mathfrak{n}_{\mathfrak{p}}}^{\wedge}$ by Corollary 5 (iv) and the first statement of this lemma. Since $M_{\mathfrak{n}_{\mathfrak{p}}}$ is finitely presented over $R_{\mathfrak{n}_{\mathfrak{p}}}$ we have $M_{\mathfrak{n}_{\mathfrak{p}}}^{\wedge}=M_{\mathfrak{n}_{\mathfrak{p}}} \otimes_{R_{\mathfrak{n}_{\mathfrak{p}}}} R_{\mathfrak{n}_{\mathfrak{p}}}^{\wedge}$, which implies the result.

3 . This follows from Corollary 6 .

Over maximal ideals the fibre and the completed fibre coincide:

Lemma 16. Let $A$ be Noetherian and $M$ a finitely generated $R$-module. For $\mathfrak{a} \subset A$ a maximal ideal the following holds.

$$
\hat{M}(\mathfrak{a})=M(\mathfrak{a}), \quad \hat{d}_{\mathfrak{a}}(M)=d_{\mathfrak{a}}(M) .
$$

(ii) $R / \mathfrak{a} R=R(\mathfrak{a})=\hat{R}(\mathfrak{a})=k(\mathfrak{a})[[x]]$ and $\mathfrak{a} R$ is a prime ideal in $R$.

(iii) $\mathfrak{n}_{\mathfrak{a}}$ is a maximal ideal of $R$ and any maximal ideal of $R$ is of the form $\mathfrak{n}_{\mathfrak{a}}$ for some $\mathfrak{a} \in \operatorname{Max} A$. Hence $\langle x\rangle$ is contained in the Jacobson radical of $R$.

(iv) $M(\mathfrak{a})=M / \mathfrak{a} M \cong M_{\mathfrak{n}_{\mathfrak{a}}} / \mathfrak{a} M_{\mathfrak{n}_{\mathfrak{a}}}$.

Proof. (i) Since $\mathfrak{a}$ is maximal, $k(\mathfrak{a})=A / \mathfrak{a}$ is a finite $A$-module. Corollary 5 (iv) implies $\hat{M}(\mathfrak{a})=M \hat{\otimes}_{A} A / \mathfrak{a}=M \otimes_{A} A / \mathfrak{a}=M(\mathfrak{a})$.

(ii) This follows from (i) and the fact that $R / \mathfrak{a} R=k(\mathfrak{a})[[x]]$ is integral.

(iii) Cf. [Mat86, §1, Example 1] and [AM69, Chapter 1, Exercise 5]). 
(iv) $M / \mathfrak{a} M=M \otimes_{A} A / \mathfrak{a}=M(\mathfrak{a})=\hat{M}(\mathfrak{a})=M \hat{\otimes}_{A} A / \mathfrak{a}=\operatorname{coker}\left(R_{\mathfrak{n}_{\mathfrak{a}}}^{p} \hat{\otimes}_{A} A / \mathfrak{a} \rightarrow\right.$ $\left.R_{\mathfrak{n}_{\mathfrak{a}}}^{q} \hat{\otimes}_{A} A / \mathfrak{a}\right)=M_{\mathfrak{n}_{\mathfrak{a}}} / \mathfrak{a} M_{\mathfrak{n}_{\mathfrak{a}}}$, as in the proof of Lemma 15 .

As a first step to semicontinuity we show below (Lemma 18) that the vanishing locus of $\hat{d}_{\mathfrak{p}}(M)$ is open. For an arbitrary $A$-module $M$

$$
\operatorname{Supp}_{A}(M):=\left\{\mathfrak{p} \in \operatorname{Spec} A \mid M_{\mathfrak{p}} \neq 0\right\}
$$

denotes the support of $M$ and $\operatorname{Ann}_{A}(M)=\{f \in A \mid f M=0\}$ the annihilator ideal of $M$.

In general $\operatorname{Supp}_{A}(M)$ is not closed in $\operatorname{Spec} A$, but if $M$ a finitely generated $A$-module, then it is well known that $\operatorname{Supp}_{A}(M)=V\left(\operatorname{Ann}_{A}(M)\right)$, which is closed in $\operatorname{Spec} A$. More generally we have:

Remark 17. For any $A$-module $M$ we have

$$
\operatorname{Supp}_{A}(M) \subset V\left(\operatorname{Ann}_{A}(M)\right) \text {. }
$$

If $R$ is an $A$-algebra and $M$ an $R$-module, then $\operatorname{Ann}_{A}(M)=\operatorname{Ann}_{R}(M) \cap A$. If $M$ is a finite $R$-module then

$$
\operatorname{Supp}_{A}(M)=V\left(\operatorname{Ann}_{A}(M)\right)
$$

and hence $\operatorname{Supp}_{A}(M)$ is closed in $\operatorname{Spec} A$.

To see the first claim, let $\mathfrak{p} \in \operatorname{Spec} A$. Note that $\mathfrak{p} \notin \operatorname{Supp}_{A} M \Leftrightarrow M_{\mathfrak{p}}=0 \Leftrightarrow$ $\forall m \in M \exists f \in A, f \notin \mathfrak{p}, f m=0$ and that $\mathfrak{p} \notin V\left(\operatorname{Ann}_{A}(M)\right) \Leftrightarrow \operatorname{Ann}_{A}(M) \nsubseteq \mathfrak{p} \Leftrightarrow$ $\exists f \notin \mathfrak{p}, f M=0$. Hence $\mathfrak{p} \notin V\left(\operatorname{Ann}_{A}(M)\right)$ implies $\mathfrak{p} \notin \operatorname{Supp}_{A}(M)$, i.e. $\operatorname{Supp}_{A}(M) \subset$ $V\left(\operatorname{Ann}_{A}(M)\right)$.

Now let $M$ be generated over $R$ by $m_{1}, \ldots, m_{k} \in M$. If $M_{\mathfrak{p}}=0$, choose $f_{i} \in A, f_{i} \notin \mathfrak{p}, f_{i} m_{i}=0$. Then $f=f_{1} \cdots f_{k} \notin \mathfrak{p}$ satisfies $f M=0$ and hence $f \in$ $\operatorname{Ann}_{A}(M)$. We get $\mathfrak{p} \notin V\left(\operatorname{Ann}_{A}(M)\right)$ and hence the other inclusion $\operatorname{Supp}_{A}(M) \supset$ $V\left(\operatorname{Ann}_{A}(M)\right)$.

In our situation for $R=A[[x]]$ and $M$ finitely $R$-presented we have (possibly strict) inclusions (cf. Lemma 12)

$$
\left\{\mathfrak{p} \in \operatorname{Spec} A \mid \hat{d}_{\mathfrak{p}}(M) \neq 0\right\} \subset\left\{\mathfrak{p} \mid d_{\mathfrak{p}}(M) \neq 0\right\} \subset \operatorname{Supp}_{A}(M),
$$

where the first (Lemma 18) and the last (Remark 17) sets are closed in Spec $A$, while the middle set may not be closed (Example 19.4).

Lemma 18. Let $M$ be a finitely presented $R=A[[x]]$-module.

1. We have (possibly strict) inclusions

$$
\left\{\mathfrak{p} \in \operatorname{Spec} A \mid \hat{d}_{\mathfrak{p}}(M) \neq 0\right\} \subset\left\{\mathfrak{p} \mid d_{\mathfrak{p}}(M) \neq 0\right\} \subset \operatorname{Supp}_{A}(M),
$$

where the first and the last sets are closed in $\operatorname{Spec} A$, while the middle set may not be closed. 
2. The map $\operatorname{Supp}_{R}(M) \rightarrow \operatorname{Supp}_{A}(M), \mathfrak{n} \mapsto \mathfrak{n} \cap A$, is dominant and induces a homeomorphism

$$
V(\langle x\rangle) \cap \operatorname{Supp}_{R}(M) \stackrel{\approx}{\rightarrow}\left\{\mathfrak{p} \in \operatorname{Spec} A \mid \hat{d}_{\mathfrak{p}}(M) \neq 0\right\} .
$$

Hence $\left\{\mathfrak{p} \in \operatorname{Spec} A \mid \hat{d}_{\mathfrak{p}}(M)=0\right\}$, the vanishing locus of $\hat{d}_{\mathfrak{p}}(M)$, is open in $\operatorname{Spec} A$.

3. Let $A^{\prime}=A / \operatorname{Ann}_{A}(M), R^{\prime}=A^{\prime}[[x]]$ and denote by $M^{\prime}$ the module $M$ considered as $A^{\prime}$-module. Then $M^{\prime}$ is a finitely presented $R^{\prime}$-module and for $\mathfrak{p} \in \operatorname{Spec}\left(A^{\prime}\right) \subset \operatorname{Spec}(A)$ we have $M_{\mathfrak{p}}=M_{\mathfrak{p}}^{\prime}, M(\mathfrak{p})=M^{\prime}(\mathfrak{p}), \hat{M}_{\mathfrak{p}}=\hat{M}_{\mathfrak{p}}^{\prime}$, and $\hat{M}(\mathfrak{p})=\hat{M}^{\prime}(\mathfrak{p})$. For $\mathfrak{p} \in \operatorname{Spec}(A) \backslash \operatorname{Spec}\left(A^{\prime}\right)$ the modules $M_{\mathfrak{p}}, M(\mathfrak{p}), \hat{M}_{\mathfrak{p}}$, and $\hat{M}(\mathfrak{p})$ vanish.

In particular, we may consider $M$ as an $A^{\prime}$-module, whenever we study the fibres or the completed fibres of $M$.

Proof. 1. The first inclusion follows from Lemma 12(i), the second from the definition. For an example where these inclusions are strict, see Example 19.2, 3 and 19.4. The first set is closed by item 2. and the third by Remark 17. In Example 19.4 the middle set is not closed.

2. Since $\operatorname{Ann}_{A}(M)=\operatorname{Ann}_{R}(M) \cap A$, the map $A / \operatorname{Ann}_{A}(M) \rightarrow R / \operatorname{Ann}_{R}(M)$ is injective and induces therefore a dominant morphism $\operatorname{Spec}\left(R / \operatorname{Ann}_{R}(M)\right) \rightarrow$ $\operatorname{Spec}\left(A / \operatorname{Ann}_{A}(M)\right)$. The first claim follows hence from Remark 17 .

For the second claim consider $A[[x]]^{p} \stackrel{T}{\rightarrow} A[[x]]^{q} \rightarrow M \rightarrow 0$, a presentation of $M$ with $T=\left(t_{i j}\right), t_{i j} \in A[[x]]$, and let $\mathfrak{p} \in \operatorname{Spec} A$. Then $k(\mathfrak{p})[[x]]^{p} \stackrel{T^{\prime}}{\rightarrow} k(\mathfrak{p})[[x]]^{q} \rightarrow$ $\hat{M}(\mathfrak{p}) \rightarrow 0$ is a presentation of $\hat{M}(\mathfrak{p})$ with $T^{\prime}=\left(t_{i j}^{\prime}\right), t_{i j}^{\prime} \in k(\mathfrak{p})[[x]]$, the induced map (Corollary 6).

Now $\hat{M}(\mathfrak{p})=0$ iff $T^{\prime}$ is surjective, i.e., iff the 0-th Fitting ideal (the ideal of $q$-minors) of $T^{\prime}$ contains a unit $u^{\prime} \in k(\mathfrak{p})[[x]]$. Write $u^{\prime}$ as $u^{\prime}=u_{0}^{\prime}+u_{1}^{\prime}$ with $u_{0}^{\prime} \in$ $k(\mathfrak{p}) \backslash\{0\}, u_{1}^{\prime} \in\langle x\rangle k(\mathfrak{p})[[x]]$. Since Fitting ideals are compatible with base change, the 0 -th Fitting ideal $F_{0} \subset A[[x]]$ of $M$ contains an element $u=u_{0}+u_{1} \in A[[x]]$ with $u_{0} \in A, u_{1} \in\langle x\rangle A[[x]]$, that maps to $u^{\prime}$ under $A[[x]] \rightarrow A_{\mathfrak{p}}[[x]] \rightarrow A_{\mathfrak{p}} / \mathfrak{p} A_{\mathfrak{p}}[[x]]$. Hence $u^{\prime}$ is a unit iff $u_{0} \notin \mathfrak{p}$, i.e., iff $\langle x, \mathfrak{p}\rangle \notin V\left(F_{0}+\langle x\rangle\right)$. The result follows since $\operatorname{Supp}_{R}(M)=V\left(F_{0}\right)$.

3. Any $R$-presentation of $M$ induces obviously an $R^{\prime}$-presentation of $M^{\prime}$. The equalities $M_{\mathfrak{p}}=M_{\mathfrak{p}}^{\prime}$ and $M(\mathfrak{p})=M^{\prime}(\mathfrak{p})$ for $\mathfrak{p} \in \operatorname{Spec}\left(A^{\prime}\right)$ are clear, the equalities $\hat{M}_{\mathfrak{p}}=\hat{M}_{\mathfrak{p}}^{\prime}$ and $\hat{M}(\mathfrak{p})=\hat{M}^{\prime}(\mathfrak{p})$ follow from this and from Lemma 12(i). Since $\operatorname{Supp}_{A}(M)=\operatorname{Spec}\left(A^{\prime}\right)$ by Remark $17, M_{\mathfrak{p}}=M(\mathfrak{p})=0$ for $\mathfrak{p} \notin \operatorname{Spec}\left(A^{\prime}\right)$ and Lemma 12(i) implies then $\hat{M}_{\mathfrak{p}}=\hat{M}(\mathfrak{p})=0$.

At the end of this section we give two examples where $d_{\mathfrak{p}}(M)$ is not semicontinuous on $\operatorname{Spec} A$ while $\hat{d}_{\mathfrak{p}}(M)$ is. The examples show also that $\hat{M}(\mathfrak{p})=0$ may happen for $M(\mathfrak{p}) \neq 0$.

Example 19. Let $A=K[t], K$ an algebraically closed field, $R=A[[x]]$, and $M=$ $R /\langle t-x\rangle \cong K[[t]]$ as $A$-module via $f(x, t) \mapsto f(t, t)$, with $t$ and $x$ one variable 
each. The following properties illustrate the difference between the fibres and the completed fibres. Let $\mathfrak{a}=\langle t-c\rangle, c \in K$, denote the maximal ideals in $A$. By Lemma $16 \hat{M}(\mathfrak{a})=M(\mathfrak{a})=M / \mathfrak{a} M$ which is isomorphic to $K[[t]] /\langle t-c\rangle$. Hence $M(\langle t\rangle)=K$ and $M(\langle t-c\rangle)=0$ for $c \neq 0$.

1. $M$ is not finitely generated over $A, d_{\mathfrak{a}}(M)=\hat{d}_{\mathfrak{a}}(M)<\infty$ for $\mathfrak{a} \in \operatorname{Max} A$ and $d_{\mathfrak{a}}(M)$ is semicontinuous on $\operatorname{Max} A$ :

$K[[t]]$ is not finite over $K[t]$ and $d_{\mathfrak{a}}(M)=1$ if $c=0$ and 0 if $c \neq 0$.

2. $d_{\mathfrak{p}}(M)$ is not semicontinuous on $\operatorname{Spec} A$ :

The prime ideal $\langle 0\rangle$ is contained in every neighbourhood of $\mathfrak{a}=\langle t\rangle$ in $\operatorname{Spec} A$. It satisfies $k(\langle 0\rangle)=K(t)$ and we get $M(\langle 0\rangle) \cong K[[t]] \otimes_{A} K(t)=K((t))$, the field of formal Laurent series. Since $\operatorname{dim}_{K(t)} K((t))=\infty, d_{\langle 0\rangle}(M)=\infty$, while $d_{\mathfrak{a}}(M) \leq 1$ for $\mathfrak{a} \in \operatorname{Spec} A \backslash\langle 0\rangle$.

3. $\hat{d}_{\mathfrak{p}}(M)$ is semicontinuous on $\operatorname{Spec} A$ :

We have $\hat{M}(\langle 0\rangle)=K(t)[[x]] /\langle t-x\rangle$ by Corollary 6 . Since $t$ is a unit in $K(t)$, $\hat{d}_{\langle 0\rangle}(M)=0$.

4. $M(\mathfrak{a})=M_{\mathfrak{a}} / \mathfrak{a} M_{\mathfrak{a}}=0$ does not imply $M_{\mathfrak{a}}=0$ :

In fact, we have $M_{\langle t-c\rangle} \cong K[[t]]_{\langle t-c\rangle}$ as a $K[t]$-module. For $c \neq 0$ we get $K[[t]]_{\langle t-c\rangle}=K((t))($ since $t \notin\langle t-c\rangle)$ while $K[[t]]_{\langle t-c\rangle} /\langle t-c\rangle K[[t]]_{\langle t-c\rangle}=$ $K((t)) /\langle t-c\rangle=0$. We have $\left\{\mathfrak{p} \mid \hat{d}_{\mathfrak{p}}(M) \neq 0\right\}=\{\langle t\rangle\} \varsubsetneqq\left\{\mathfrak{p} \mid d_{\mathfrak{p}}(M) \neq 0\right\}=$ $\{\langle t\rangle,\langle 0\rangle\} \varsubsetneqq \operatorname{Supp}_{A}(M)=\operatorname{Spec} A$.

5. $M$ is flat over $A$. By 1 . and 3 . we cannot expect any continuity of $d_{\mathfrak{p}}(M)$ or $\hat{d}_{\mathfrak{p}}(M)$ on $\operatorname{Max} A$ or on $\operatorname{Spec} A$ for flat $A$-modules.

6. The quasi-finite locus of $A \rightarrow A[[x]] /\langle t-x\rangle$ is not open in Spec $A$ :

The quasi-finite locus $\left\{\mathfrak{p} \in \operatorname{Spec} A \mid d_{\mathfrak{p}}(M)<\infty\right\}$ is $\operatorname{Spec} A \backslash\langle 0\rangle$ by 1 . and 2 . Recall that if $B$ is a ring of finite type over $A$, then the quasi-finite locus of $A \rightarrow B$ is open by Zariski's main Theorem (cf. [Sta19, 10.122]).

7. The quasi-completed-finite locus of $A \rightarrow A[[x]] /\langle t-x\rangle$ is open in Spec $A$ :

Let us call $\left\{\mathfrak{p} \in \operatorname{Spec} A \mid \hat{d}_{\mathfrak{p}}(M)<\infty\right\}$ the quasi-completed-finite locus. It is $\operatorname{Spec} A$ in our example.

In general, if semicontinuity of $\hat{d}_{\mathfrak{p}}(M)$ holds (Corollary 45$)$, then the quasicompleted-finite locus is open.

Example 20. The following example may be of interest for arithmetic and computational purposes. It goes along similar lines as Example 19.

Let $A=\mathbb{Z}, R=\mathbb{Z}[[x]]$, and $M=R /\langle x-p\rangle, p \in \mathbb{Z}$ a prime number. Since $R=\lim _{\longleftarrow} \mathbb{Z}[x] /\langle x\rangle^{n}$ we obtain $M=\lim _{\longleftarrow} \mathbb{Z} / p^{n}=\hat{\mathbb{Z}}_{\langle p\rangle}$, the ring of $p$-adic integers.

Now let $\langle q\rangle \in \operatorname{Max} \mathbb{Z}$.

If $q \neq p$ then $q$ is a unit in $\mathbb{Z}_{\langle p\rangle}$ hence in $\hat{\mathbb{Z}}_{\langle p\rangle}$ and $M \otimes_{\mathbb{Z}} \mathbb{Z} / q=M /\langle q\rangle M=$ $\hat{\mathbb{Z}}_{\langle p\rangle} / q \hat{\mathbb{Z}}_{\langle p\rangle}=0$.

If $q=p$ then $M \otimes_{\mathbb{Z}} \mathbb{Z} / p=\hat{\mathbb{Z}}_{\langle p\rangle} / p \hat{\mathbb{Z}}_{\langle p\rangle}=\mathbb{Z} / p$.

Hence $d_{\langle q\rangle}(M)=\operatorname{dim}_{\mathbb{Z} / q} M \otimes_{\mathbb{Z}} \mathbb{Z} / q$ is 0 if $q \neq p$ and 1 if $q=p$. 
On the other hand, looking at the prime ideal $\langle 0\rangle$ we get

$$
\hat{M}(\langle 0\rangle)=M \hat{\otimes}_{\mathbb{Z}} \mathbb{Q}=\mathbb{Q}[[x]] /\langle x-p\rangle=0,
$$

while

$$
M(\langle 0\rangle)=M \otimes_{\mathbb{Z}} \mathbb{Q}=\hat{\mathbb{Z}}_{\langle p\rangle} \otimes_{\mathbb{Z}} \mathbb{Q}=\operatorname{Quot}\left(\hat{\mathbb{Z}}_{\langle p\rangle}\right)
$$

has dimension $d_{\langle 0\rangle}(M)=\operatorname{dim}_{\mathbb{Q}} \operatorname{Quot}\left(\hat{\mathbb{Z}}_{\langle p\rangle}\right)=\infty$.

To see the last equality in the formula for $M(\langle 0\rangle)$ one checks that the following diagram has the universal property of the tensor product:

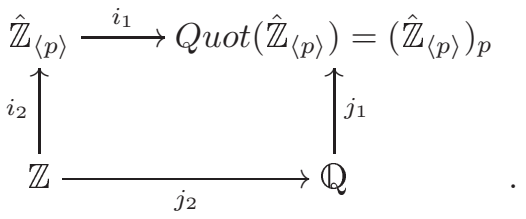

Here $i_{1}, i_{2}$ and $j_{2}$ are the canonical inclusions and $j_{1}$ is given as follows: if $\alpha, \beta \in \mathbb{Z}$, $p \nmid \beta$, then $j_{1}\left(\frac{\alpha}{p^{m} \beta}\right)=\frac{1}{p^{m}} \frac{\alpha}{\beta}, \frac{\alpha}{\beta} \in \hat{\mathbb{Z}}_{\langle p\rangle}$ since $p \nmid \beta$. The universality of the diagram is easily seen. If $T$ is a $\mathbb{Z}-$ algebra and $\phi: \hat{\mathbb{Z}}_{\langle p\rangle} \rightarrow T$ and $\psi: \mathbb{Q} \rightarrow \mathbb{Z}$ are $\mathbb{Z}$ algebra homomorphisms then the morphism $\sigma:\left(\hat{\mathbb{Z}}_{\langle p\rangle}\right)_{p} \rightarrow T$, given as $\sigma\left(\alpha / p^{m}\right)=$ $\phi(\alpha) \psi\left(1 / p^{m}\right), p \nmid \alpha$ is the unique one, making the obvious diagram commutative.

\subsection{Semicontinuity over a 1-dimensional ring}

In this section $A$ will be Noetherian and $M$ a finitely generated $R$-module (unless we say otherwise). Then $R=A[[x]]$ is Noetherian and $M$ is finitely presented as $R$-module. At the moment we can prove the semicontinuity of $d_{\mathfrak{q}}(M)$ on Spec $A$ in full generality only under certain assumptions on the irreducible components of $\operatorname{Supp}_{R}(M)$. This includes the case $\operatorname{dim} A=1$ where $\operatorname{dim} A$ denotes the Krull dimension of $A$. The case of arbitrary Noetherain $A$ is treated in the next section under the assumption that the presentation matrix of $M$ is algebraic.

In an important special case semicontinuity holds for arbitrary $A$ :

Proposition 21. Let $A$ be Noetherian and $M$ a finitely generated $R$-module.

1. If $\operatorname{Supp}_{R}(M) \subset V(\langle x\rangle)$ then $M$ is finitely generated over $A$ and $\hat{M}(\mathfrak{q})=$ $M(\mathfrak{q})$ for all $\mathfrak{q} \in \operatorname{Spec} A$. In particular semicontinuity of $\hat{d}_{\mathfrak{p}}(M)=d_{\mathfrak{p}}(M)$ holds at any $\mathfrak{p} \in \operatorname{Spec} A$.

2. If $M$ is finitely generated as an $A$-module (in particular $d_{\mathfrak{p}}(M)<\infty$ for $\mathfrak{p} \in \operatorname{Spec} A$ ), then $\hat{d}_{\mathfrak{p}}(M) \leq d_{\mathfrak{p}}(M)$ and $\hat{d}_{\mathfrak{p}}(M)$ (as well as $d_{\mathfrak{p}}(M)$ ) is semicontinuous at any $\mathfrak{p} \in \operatorname{Spec} A$.

Proof. 1. Since $V\left(\operatorname{Ann}_{R}(M)\right)=\operatorname{Supp}_{R}(M) \subset V(\langle x\rangle)$, we have $\langle x\rangle \subset \sqrt{\operatorname{Ann}_{R}(M)}$ and there exists an $m$ such that $\langle x\rangle^{m} \subset \operatorname{Ann}_{R}(M)$. We get a surjection

$$
A[[x]] /\langle x\rangle^{m} \rightarrow R / \operatorname{Ann}_{R}(M)
$$


and since $A[[x]] /\langle x\rangle^{m}$ is finitely generated over $A$ this holds for $R / \operatorname{Ann}_{R}(M)$ too. Since $M$ is finitely generated over $R / \operatorname{Ann}_{R}(M)$ it is finitely generated over $A$ and hence finitely presented. By Lemma 1 there there is an open neighborhood $U$ of $\mathfrak{p}$ in Spec $A$ such that $d_{\mathfrak{q}}(M) \leq d_{\mathfrak{p}}(M), \mathfrak{q} \in U$. By Proposition 3.5, $\hat{M}(\mathfrak{q})=M(\mathfrak{q})$ for all $\mathfrak{q} \in \operatorname{Spec} A$, showing the claim.

2. Let $m<m^{\prime}$ be two strictly positive integers and consider the natural surjective maps

$$
R \longrightarrow R /\langle x\rangle^{m^{\prime}} \longrightarrow R /\langle x\rangle^{m} .
$$

By the right exactness of the tensor product, they induce surjective maps

$$
M \longrightarrow M /\langle x\rangle^{m^{\prime}} M \longrightarrow M /\langle x\rangle^{m} M
$$

and

$$
M(\mathfrak{p})=M \otimes_{A} k(\mathfrak{p}) \longrightarrow M /\langle x\rangle^{m^{\prime}} M \otimes_{A} k(\mathfrak{p}) \stackrel{\pi_{m, m^{\prime}}(\mathfrak{p})}{\longrightarrow} M /\langle x\rangle^{m} M \otimes_{A} k(\mathfrak{p}) .
$$

Since $M$ is finitely generated over $A$, all the modules appearing in the last sequence are finite-dimensional $k(\mathfrak{p})$-vector spaces, with $\operatorname{dim}_{k(\mathfrak{p})}(M(\mathfrak{p}))=d_{\mathfrak{p}}(M)$ by definition. Since $\pi_{m, m^{\prime}}(\mathfrak{p})$ is surjective for all $m<m^{\prime}$, the elements of the inverse system $\left\{M /\langle x\rangle^{m} M \otimes_{A} k(\mathfrak{p})\right\}_{m}$ of finite-dimensional $k(\mathfrak{p})$-vector spaces have dimensions increasing with $m$ and bounded above by $d_{\mathfrak{p}}(M)$. Thus $\operatorname{dim}_{k(\mathfrak{p})}\left(M /\langle x\rangle^{m} M \otimes_{A} k(\mathfrak{p})\right)$ stabilizes for large $m$. Hence, for $m$ large

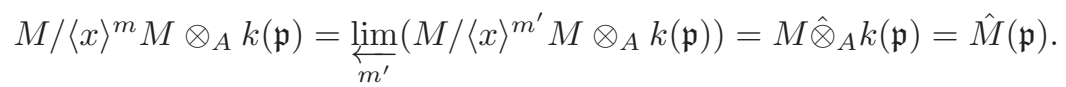

This implies $\hat{d}_{\mathfrak{p}}(M)=\operatorname{dim}_{k(\mathfrak{p})} \hat{M}(\mathfrak{p}) \leq d_{\mathfrak{p}}(M)$.

To see the semicontinuity of $\hat{d}_{\mathfrak{p}}(M)$, we use the semicontinuity of $d_{\mathfrak{p}}(M)$ (by Lemma 1). It follows that there exists an open neighbourhood $U$ of $\mathfrak{p}$ such that the sequence $\left\{\operatorname{dim}_{k(\mathfrak{q})}\left(M /\langle x\rangle^{m} M \otimes_{A} k(\mathfrak{q})\right)\right\}_{m}$ is bounded by $d_{\mathfrak{p}}(M)$ simultaneously for all $\mathfrak{q} \in U$. Hence,

$$
d_{\mathfrak{q}}\left(M /\langle x\rangle^{m} M\right)=\operatorname{dim}_{k(\mathfrak{q})}\left(M /\langle x\rangle^{m} M \otimes_{A} k(\mathfrak{q})\right)=\operatorname{dim}_{k(\mathfrak{q})}(\hat{M}(\mathfrak{q}))=\hat{d}_{\mathfrak{q}}(M)
$$

for a fixed large $m$ and $\mathfrak{q} \in U$. Since $M /\langle x\rangle^{m} M$ is finitely $A$-generated, Lemma 1 implies that $d_{\mathfrak{p}}\left(M /\langle x\rangle^{m} M\right)$ is semicontinuous, and so is $\hat{d}_{\mathfrak{p}}(M)$.

The inequality $\hat{d}_{\mathfrak{p}}(M) \leq d_{\mathfrak{p}}(M)$ of item 2. and its proof, as well as Example 49 , were suggested to us by the anonymous referee. Note that $\hat{d}_{\mathfrak{p}}(M)=d_{\mathfrak{p}}(M)$ for $\mathfrak{p}$ a maximal ideal (by Lemma 16), but that $\hat{d}_{\mathfrak{p}}(M)<d_{\mathfrak{p}}(M)$ may happen by Example 50 for $\mathfrak{p}$ not maximal.

Before we formulate the next result, we introduce some notations to be used throughout this section. Consider a minimal primary decomposition of $\operatorname{Ann}_{R}(M)$,

$$
\operatorname{Ann}_{R}(M)=\bigcap_{i=1}^{r} Q_{i} \subset R .
$$


Since $M$ is finitely generated over $R, \operatorname{Supp}_{R}(M)=V\left(\operatorname{Ann}_{R}(M)\right)=\cup_{i=1}^{r} V\left(Q_{i}\right)$ and $\operatorname{dim} M=\operatorname{dim} \operatorname{Supp}_{R}(M)$.

Let $P_{1}, \ldots P_{s} \subset R$ be the minimal associated primes of $\langle x\rangle$. Since they correspond via $h: A \cong R /\langle x\rangle$ to the minimal associated primes $\bar{P}_{1}, \ldots \bar{P}_{s}$ of $A$, we have $\operatorname{dim} V\left(P_{j}\right) \leq \operatorname{dim} A$.

Lemma 22. For $A$ Noetherian, $M$ finitely generated over $R$ and $\mathfrak{p} \in \operatorname{Spec} A$ the following holds:

1. Let $A^{\prime}$ be the reduction of $A, R^{\prime}=A^{\prime}[[x]]$ and $M^{\prime}$ the $R^{\prime}$-module $M \otimes_{R} R^{\prime}$. Then $\hat{M}^{\prime}(\mathfrak{p}) \cong \hat{M}(\mathfrak{p})$ and hence $\hat{d}_{\mathfrak{p}}\left(M^{\prime}\right)=\hat{d}_{\mathfrak{p}}(M)$.

2. Let $Q \subset R$ be an ideal. Then $\hat{d}_{\mathfrak{p}}(M / Q M) \leq \hat{d}_{\mathfrak{p}}(M)$.

3. If $Q_{i} \not \subset \mathfrak{n}_{\mathfrak{p}}$ for some $1 \leq i \leq r$, then $\hat{d}_{\mathfrak{q}}(M)=\hat{d}_{\mathfrak{q}}(M / Q M)$, with $Q=\cap_{j \neq i} Q_{j}$, for $\mathfrak{q}$ in some neighbourhood of $\mathfrak{p}$ in $\operatorname{Spec} A$.

4. If $Q_{i} \subset \mathfrak{n}_{\mathfrak{p}}$ and $\operatorname{dim} V\left(Q_{i}\right)>\operatorname{dim}\left(A / Q_{i} \cap A\right)_{\mathfrak{p}}$ for some $1 \leq i \leq r$, then $\hat{d}_{\mathfrak{p}}(M)=\infty$.

5. Let $U=\operatorname{Spec} B \subset \operatorname{Spec} A$ be an affine open neighbourhood of $\mathfrak{p}$ and $M_{B}=$ $M \otimes_{A} B$ the restriction of $M$ to $U$. Then $\hat{M}_{B}(\mathfrak{q})=\hat{M}(\mathfrak{q})$ for all $\mathfrak{q} \in U$.

Proof. 1. Since $\mathfrak{p} \in \operatorname{Spec} A$ contains the nilpotent elements, $A^{\prime} / \mathfrak{p}^{\prime}=A / \mathfrak{p}$, where $\mathfrak{p}^{\prime}$ is the image of $\mathfrak{p}$ in $A^{\prime}$, and hence the residue field does not change if we pass from $A$ to $A^{\prime}$. By Proposition 3.1 we have $\hat{R}^{\prime}\left(\mathfrak{p}^{\prime}\right)=R^{\prime} \hat{\otimes}_{A^{\prime}} k(\mathfrak{p})=k(\mathfrak{p})[[x]]=\hat{R}(\mathfrak{p})$. Consider a presentation $R^{p} \stackrel{T}{\rightarrow} R^{q} \rightarrow M \rightarrow 0$ of $M$. Applying $\otimes_{R} R^{\prime}$, we get a presentation of $M^{\prime}, R^{\prime p} \stackrel{T}{\rightarrow} R^{\prime q} \rightarrow M^{\prime} \rightarrow 0$. Apply $\hat{\otimes}_{A} k(\mathfrak{p})$ to the first resp. $\hat{\otimes}_{A^{\prime}} k(\mathfrak{p})$ to the second exact sequence above. The sequences stay exact by Corollary 4 . Since $(\hat{R}(\mathfrak{p}))^{k}=\left(\hat{R}^{\prime}\left(\mathfrak{p}^{\prime}\right)\right)^{k}$ it follows that the canonical morphism $M \rightarrow M^{\prime}$ induces an isomorphism $\hat{M}(\mathfrak{p}) \cong \hat{M}^{\prime}(\mathfrak{p})$.

2. Since $(M / Q M) \hat{\otimes}_{A} k(\mathfrak{p})=M \hat{\otimes}_{A} k(\mathfrak{p}) / Q\left(M \hat{\otimes}_{A} k(\mathfrak{p})\right)$ by Corollary 4, the result follows.

3. $Q_{i} \not \subset \mathfrak{n}_{\mathfrak{p}}$ means that $\mathfrak{n}_{\mathfrak{p}}$ is not a point of $V\left(Q_{i}\right)$. Hence $\mathfrak{n}_{\mathfrak{q}} \notin V\left(Q_{i}\right)$ and $M_{\mathfrak{n}_{\mathfrak{q}}}=(M / Q)_{\mathfrak{n}_{\mathfrak{q}}}$ for $\mathfrak{n}_{\mathfrak{q}}$ in some neighbourhood of $\mathfrak{n}_{\mathfrak{p}}$ in $V(\langle x\rangle)$. The result follows from Lemma 15.

4. Set $\bar{R}:=R / Q_{i}, \bar{A}:=A / Q_{i} \cap A$ and $\bar{M}:=M / Q_{i} M$. Then $Q_{i} \subset \operatorname{Ann}_{R}(\bar{M}) \subset$ $\sqrt{\left(Q_{i}\right)}$ and $\operatorname{dim} \bar{R}_{\mathfrak{n}_{\mathfrak{p}}}=\operatorname{dim} \bar{M}_{\mathfrak{n}_{\mathfrak{p}}}=\operatorname{dim} V\left(Q_{i}\right)>\operatorname{dim} \bar{A}_{\mathfrak{p}}$ by assumption. Considering $\bar{M}$ as $R$-module, we have $\bar{M} \hat{\otimes}_{A} k(\mathfrak{p})=\bar{M}(\mathfrak{p})^{\wedge}=\left(\bar{M}_{\mathfrak{n}_{\mathfrak{p}}} / \mathfrak{p} \bar{M}_{\mathfrak{n}_{\mathfrak{p}}}\right)^{\wedge}$ by Lemma 15. Since the $\bar{R}_{\mathfrak{n}_{\mathfrak{p}}}$-modules $\bar{M}(\mathfrak{p})$ and its $\langle x\rangle$-adic completion $\bar{M}(\mathfrak{p})^{\wedge}$ have the same Hilbert-Samuel function w.r.t. $\mathfrak{n}_{\mathfrak{p}}$, their dimension coincides (c.f. [GP08, Corollary 5.6.6]). Moreover, $\mathfrak{p} \bar{R}_{\mathfrak{n}_{\mathfrak{p}}}$ is the annihilator of $\bar{M}_{\mathfrak{n}_{\mathfrak{p}}} / \mathfrak{p} \bar{M}_{\mathfrak{n}_{\mathfrak{p}}}$ and therefore $\operatorname{dim} \bar{M}(\mathfrak{p})^{\wedge}=\operatorname{dim} \bar{R}_{\mathfrak{n}_{\mathfrak{p}}} / \mathfrak{p} \bar{R}_{\mathfrak{n}_{\mathfrak{p}}}$.

We apply now [Mat86, Theorem 15.1] to the map of local rings $\bar{A}_{\mathfrak{p}} \rightarrow \bar{R}_{\mathfrak{n}_{\mathfrak{p}}}$ and get that $\operatorname{dim} \bar{R}_{\mathfrak{n}_{\mathfrak{p}}} / \mathfrak{p} \bar{R}_{\mathfrak{n}_{\mathfrak{p}}} \geq \operatorname{dim} \bar{R}_{\mathfrak{n}_{\mathfrak{p}}}-\operatorname{dim} \bar{A}_{\mathfrak{p}}>0$ and hence $\operatorname{dim}_{k(\mathfrak{p})} \bar{M}(\mathfrak{p})^{\wedge}=\infty$. Then $\hat{d}_{\mathfrak{p}}(M)=\operatorname{dim}_{k(\mathfrak{p})} M(\mathfrak{p})^{\wedge}=\infty$ by 2 . of this lemma. 
5. We may assume that $B=A_{f}$ for some $f \notin \mathfrak{p}$. Since $A_{\mathfrak{q}}=\left(A_{f}\right)_{\mathfrak{q}}$ for $\mathfrak{q} \in U=D(f)$, we have $k(\mathfrak{q})=A_{f} \otimes_{A} k(\mathfrak{q})$. Now Proposition 3.1 implies $\hat{M}_{A_{f}}(\mathfrak{q})=$ $\left(M \otimes_{A} A_{f}\right) \hat{\otimes}_{A} k(\mathfrak{q})=\left(M \otimes_{A} A_{f} \otimes_{A} k(\mathfrak{q})\right)^{\wedge}=M(\mathfrak{q})^{\wedge}=\hat{M}(\mathfrak{q})$.

Proposition 23. Let $A$ be Noetherian, $M$ a finitely generated $R$-module and fix $\mathfrak{p} \in \operatorname{Spec} A$. Let $Q_{1}, \ldots, Q_{r}$ be the primary components of $A n n_{R}(M)$, which we renumerate such that

I. $V\left(Q_{i}\right) \subset V(\langle x\rangle)$ for $1 \leq i \leq k$,

II. $V\left(Q_{i}\right) \not \subset V(\langle x\rangle)$ for $k+1 \leq i \leq r$,

and set $Q_{I}:=\bigcap_{i=1}^{k} Q_{i}$ and $Q_{I I}:=\stackrel{h}{r}_{i=k+1} Q_{i}$. Assume that either (a) $V\left(Q_{I I}\right)=\emptyset$ (i.e., $k=r$ ), or (b) $\operatorname{dim} V\left(Q_{I I}\right)>\operatorname{dim} V\left(Q_{I I} \cap A\right)$ or $(c) \mathfrak{n}_{\mathfrak{p}}$ is an isolated point of $V(\langle x\rangle) \cap V\left(Q_{I I}\right)$.

Then there is an open neighbourhood $U$ of $\mathfrak{p}$ in $\operatorname{Spec} A$ such that $\hat{d}_{\mathfrak{q}}(M) \leq$ $\hat{d}_{\mathfrak{p}}(M)$ for all prime ideals $\mathfrak{q} \in U$.

Proof. We set $M_{I}:=M / Q_{I} M$ and $M_{I I}:=M / Q_{I I} M$. Then $\operatorname{Ann}_{R}\left(M_{I}\right)=Q_{I}$ and $\operatorname{Ann}_{R}\left(M_{I I}\right)=Q_{I I}$. By Lemma 22.3 we may assume that $Q_{i} \subset \mathfrak{n}_{\mathfrak{p}}$ for all $1 \leq i \leq r$.

We have $\operatorname{Supp}_{R}\left(M_{I}\right)=V\left(Q_{I}\right) \subset V(\langle x\rangle)$. By Proposition 21 there is an open neighborhood $U_{1}$ of $\mathfrak{p}$ in Spec $A$ such that

$$
\hat{d}_{\mathfrak{q}}\left(M_{I}\right) \leq \hat{d}_{\mathfrak{p}}\left(M_{I}\right), \mathfrak{q} \in U_{1} .
$$

(a) If $V\left(Q_{I I}\right)=\emptyset$, then $M=M_{I}$ and the claim follows from (1).

(b) If $\operatorname{dim} V\left(Q_{I I}\right)>\operatorname{dim} V\left(Q_{I I} \cap A\right)$ then $\operatorname{dim} V\left(Q_{i}\right)>\operatorname{dim}\left(A / Q_{i} \cap A\right)_{\mathfrak{p}}$ for some $i$ and hence $\hat{d}_{\mathfrak{p}}(M)=\infty$ by Lemma 22.4, implying the claim.

(c) Now let $\mathfrak{n}_{\mathfrak{p}}$ be an isolated point of $V(\langle x\rangle) \cap V\left(Q_{I I}\right)$. Then there exists an open neighbourhood $U_{2} \subset \operatorname{Spec} A$ of $\mathfrak{p}$ such that $M_{I, \mathfrak{n}_{\mathfrak{q}}}=M_{\mathfrak{n}_{\mathfrak{q}}}$ if $\mathfrak{q} \in U_{2} \backslash\{\mathfrak{p}\}$. Since $\hat{d}_{\mathfrak{q}}(M)=\operatorname{dim}_{\mathfrak{k}}\left(M_{\mathfrak{n}_{\mathfrak{q}}} / \mathfrak{q} M_{\mathfrak{n}_{\mathfrak{q}}}\right)^{\wedge}$ we get

$$
\hat{d}_{\mathfrak{q}}(M)=\hat{d}_{\mathfrak{q}}\left(M_{I}\right), \mathfrak{q} \in U_{2} \backslash\{\mathfrak{p}\} .
$$

Using (1) and (2), we have $\hat{d}_{\mathfrak{q}}(M) \leq \hat{d}_{\mathfrak{p}}\left(M_{I}\right)$ for $\mathfrak{q} \in U_{1} \cap U_{2} \backslash\{\mathfrak{p}\}$ and by Lemma 22.2

$$
\hat{d}_{\mathfrak{p}}\left(M_{I}\right) \leq \hat{d}_{\mathfrak{p}}(M)
$$

Hence $\hat{d}_{\mathfrak{q}}(M) \leq \hat{d}_{\mathfrak{p}}(M)$ for $\mathfrak{q} \in U_{1} \cap U_{2}$.

As a corollary we get the following theorem, which was already proved for maximal ideals in $A=\mathbb{k}[t]$ in [GPh19].

Theorem 24. Let $A$ be Noetherian, $M$ a finitely generated $R$-module and $\mathfrak{p} \in$ $\operatorname{Spec} A$. If $\operatorname{dim}_{\mathfrak{p}}\left(\operatorname{Supp}_{A}(M)\right) \leq 1$ then there is an open neighbourhood $U$ of $\mathfrak{p}$ in Spec $A$ such that

$$
\hat{d}_{\mathfrak{q}}(M) \leq \hat{d}_{\mathfrak{p}}(M) \text { for all } \mathfrak{q} \in U .
$$


Proof. By Lemma 18.3 we may assume that $\operatorname{Ann}_{A}(M)=0$, such that $\operatorname{Supp}_{A}(M)=$ $A$. We may further assume that $\operatorname{dim} A=\operatorname{dim} V(\langle x\rangle)=1$ and $\hat{d}_{\mathfrak{p}}(M)<\infty$. Using the notations from Proposition 23, we have $\operatorname{dim} V\left(Q_{I I} \cap A\right) \leq 1$ and by the proof of Proposition 23(b) that $\operatorname{dim} V\left(Q_{I I}\right) \leq 1$. Hence, either $V\left(Q_{I I}\right)=\emptyset$, or $\mathfrak{n}_{\mathfrak{p}}$ is an isolated point of $V(\langle x\rangle) \cap V\left(Q_{I I}\right)$. The result follows from Proposition 23 .

Corollary 25. Let $A=\mathbb{Z}$ and let $M$ be a finitely generated $\mathbb{Z}[[x]]$-module, $x=$ $\left(x_{1} \cdots x_{n}\right)$, given by a presentation

$$
\mathbb{Z}[[x]]^{r} \rightarrow \mathbb{Z}[[x]]^{s} \rightarrow M \rightarrow 0 .
$$

Denote by

$$
M_{p}:=\hat{M}(\langle p\rangle)=\operatorname{coker}\left(\mathbb{F}_{p}[[x]]^{r} \stackrel{\bar{T}}{\rightarrow} \mathbb{F}_{p}[[x]]^{s}\right)
$$

if $p \in \mathbb{Z}$ is a prime number and by

$$
M_{0}:=\hat{M}(\langle 0\rangle)=\operatorname{coker}\left(\mathbb{Q}[[x]]^{r} \stackrel{\bar{T}}{\rightarrow} \mathbb{Q}[[x]]^{s}\right)
$$

the induced modules.

1. Fix a prime number $p$. If $\operatorname{dim}_{\mathbb{F}_{p}} M_{p}<\infty$ then $\operatorname{dim}_{\mathbb{F}_{p}} M_{p} \geq \operatorname{dim}_{\mathbb{Q}} M_{0}$. Moreover, for all except finitely many prime numbers $q \in \mathbb{Z}, \operatorname{dim}_{\mathbb{F}_{p}} M_{p} \geq$ $\operatorname{dim}_{\mathbb{F}_{q}} M_{q}$.

2. If $\operatorname{dim}_{\mathbb{Q}} M_{0}<\infty$ then $\operatorname{dim}_{\mathbb{Q}} M_{0} \geq \operatorname{dim}_{\mathbb{F}_{q}} M_{q}$ for all except finitely many prime numbers $q \in \mathbb{Z}$, and hence "=" for all except finitely many prime numbers $q \in \mathbb{Z}$.

The first part of statement 1 . follows, since $\langle 0\rangle$ is in every neighbourhood of $p$. In particular $\operatorname{dim}_{\mathbb{Q}} M_{0}$ is finite if $\operatorname{dim}_{\mathbb{F}_{p}} M_{p}$ is finite for some prime number $p$.

Remark 26. The corollary is important for practical computations in computer algebra systems. For simplicity let $I$ be an ideal in $\mathbb{Z}[[x]]$ generated by polynomials, $M=\mathbb{Z}[[x]] / I$, and $I_{p}$ the image of $I$ in $\mathbb{F}_{p}[[x]]$. The dimension of $\mathbb{Q}[[x]] / I$ resp. of $\mathbb{F}_{p}[[x]] / I_{p}$, if finite, is equal to the dimension of $\mathbb{Q}[x]_{\langle x\rangle} / I$ resp. of $\mathbb{F}_{p}[x]_{\langle x\rangle} / I_{p}$. These dimensions can be computed in the localizations $\mathbb{Q}[x]_{\langle x\rangle}$ resp. $\mathbb{F}_{p}[x]_{\langle x\rangle}$ by computing a Gröbner or standard basis of $I$ resp. of $I_{p}$ w.r.t. a local monomial ordering (cf. [GP08]). Such algorithms are implemented e.g. in SINGULAR [DGPS]. Usually the computations over $\mathbb{Q}$ are very time consuming or do not finish, due to extreme coefficient growths, and therefore often modular methods are used. The above corollary says that for all except finally many prime numbers $p$ we have equality $\operatorname{dim}_{\mathbb{Q}} \mathbb{Q}[x]_{\langle x\rangle} / I=\operatorname{dim}_{\mathbb{F}_{p}} \mathbb{F}_{p}[x]_{\langle x\rangle} / I_{p}$, and if this holds $p$ is sometimes called a "lucky" prime. This fact can also be proved by Gröbner basis methods. More interesting is however that $\operatorname{dim}_{\mathbb{Q}} \mathbb{Q}[x]_{\langle x\rangle} / I<\infty$ if there exists just one $p$ (lucky or not) such that $\operatorname{dim}_{\mathbb{F}_{p}} \mathbb{F}_{p}[x]_{\langle x\rangle} / I_{p}<\infty$ and that the first dimension is bounded by the latter. This was stated in [Pf07] without proof. 


\subsection{Henselian rings and Henselian tensor product}

In this section we recall some basic facts about Henselian rings and introduce similarly to the complete tensor product a Henselian tensor product. For details about Henselian rings see [Sta19] or [KPR75]. The Henselian tensor product is needed in Section 1.5 for algebraically presented modules. We start with some basic facts about étale ring maps.

\section{Definition 27.}

1. A ring $\operatorname{map} \phi: A \longrightarrow B$ is called étale if it is flat, unramified and of finite presentation. $^{2}$

2. $\phi$ is called standard étale if $B=(A[T] / F)_{G}, F, G \in A[T]$, the univariate polynomial ring, $F$ monic and $F^{\prime}$ a unit in $B$.

3. $\phi$ is called étale at $\mathfrak{q} \in \operatorname{Spec}(B)$ if there exist $g \in B \backslash \mathfrak{q}$ such that $A \longrightarrow B_{g}$ is étale.

The following proposition lists some basic properties of étale maps. The results can be found in section 10.142 of [Sta19].

\section{Proposition 28.}

1. The map $A \longrightarrow A_{f}$ is étale.

2. A standard étale map is an étale map.

3. The composition of étale maps is étale.

4. A base change of étale maps is étale.

5. An étale map is open.

6. An étale map is quasi-finite.

7. Given $\phi: A \longrightarrow B$ and $g_{1}, \ldots, g_{m} \in B$ generating the unit ideal ${ }^{3}$ such that $A \longrightarrow B_{g_{i}}$ is étale for all $i$ then $\phi: A \longrightarrow B$ is étale.

8. Let $\phi: A \longrightarrow B$ be étale. Then there exist $g_{1}, \ldots, g_{m} \in B$ generating the unit ideal such that $A \longrightarrow B_{g_{i}}$ is standard étale for all $i$.

9. Let $S \subset A$ be a multiplicatively closed subset and assume that $\phi^{\prime}: S^{-1} A \longrightarrow$ $B^{\prime}$ is étale. Then there exists an étale map $\phi: A \longrightarrow B$ such that $B^{\prime}=S^{-1} B$ and $\phi^{\prime}=S^{-1} \phi$.

10. Let $\phi^{\prime}: A / I \longrightarrow B^{\prime}$ be étale for some ideal $I \subset A$. Then there exist an étale map $\phi: A \longrightarrow B$ such that $B^{\prime}=B / I B$ and the obvious diagram commutes.

Definition 29. Let $A$ be a ring and $I \subset A$ an ideal. $A$ is called Henselian with respect to $I$ if the following holds ${ }^{4}$ (Univariate Implicit Function Theorem):

Let $F \in A[T]$, the univariate polynomial ring, such that $F(0) \in I$ and $F^{\prime}(0)$ is a unit modulo $I$. Then there exists $a \in I$ such $^{5}$ that $F(a)=0$.

${ }^{2} \phi$ is unramified if it is of finite type and the module of Kähler differentials $\Omega_{B / A}$ vanishes. $\phi$ is of finite presantation if $B \cong A\left[x_{1}, \ldots, x_{n}\right] /\left\langle f_{1}, \ldots, f_{m}\right\rangle$ as $A$-algebras.

3 i.e., $\operatorname{Spec}(A)=\cup D\left(g_{i}\right)$.

${ }^{4}$ Note, that (similarly to the $I$-adic completion) the condition implies that $I$ is contained in the Jacobson radical of $A$. If we start with an ideal contained in the Jacobson radical then it is enough to consider monic polynomials $F$ in the definition.

${ }^{5}$ Note that $a$ is uniquely determined by the condition $a \in I$, [KPR75]. 
Next we associate to any pair $(A, I), I \subset A$ an ideal, the Henselization $A_{I}^{h}$, i.e. the "smallest" Henselian ring with respect to $I$, such that $A_{I}^{h} \subset \hat{A}_{I}=\underset{\longleftarrow}{\lim }\left(A / I^{n}\right)$ the $I$-adic completion.

\section{Definition 30.}

1. Let $A$ be a ring and $I \subset A$ an ideal. The ring

$$
A_{I}^{h}=\lim _{\longrightarrow}(B \mid A \longrightarrow B \text { an étale ring map inducing } A / I \cong B / I B)
$$

is called the Henselization of $A$ with respect to $I$.

2. The Henselization of $A[x], A$ any ring, $x=\left(x_{1}, \ldots, x_{n}\right)$, with respect to $I=\langle x\rangle$ is denoted by $A\langle x\rangle$. We call $A\langle x\rangle$ the ring of albegraic power series over $A$.

The Henselization has the following properties (cf. section 15.11 and 15.12 of [Sta19]):

Proposition 31. Let $A$ be ring and $I \subset A$ an ideal.

1. $A_{I}^{h}$ is Henselian with respect to $I^{h}=I A_{I}^{h}$ and $A / I^{m}=A_{I}^{h} /\left(I^{h}\right)^{m}$ for all $m$.

2. A is Henselian with respect to $I$ if and only if $A=A_{I}^{h}$.

3. If $A$ is Noetherian then the canonical map $A \longrightarrow A_{I}^{h}$ is flat.

4. If $A$ is Noetherian then the canonical map $A_{I}^{h} \longrightarrow \hat{A}_{I}$ is faithfully flat and $\hat{A}_{I}$ is the $I^{h}$-adic completion of $A_{I}^{h}$.

Remark 32. The definition of the Henselization implies that $A_{I}^{h}$ is contained in the algebraic closure of $A$ in $\hat{A}_{I}$. If $A$ is excellent ${ }^{6}$ then $A_{I}^{h}$ is the algebraic closure of $A$ in $\hat{A}_{I}$. This is even true under milder conditions, see [KPR75]. In this situation $C\langle x\rangle$ is called the ring of algebraic power series of $C[[x]]$.

Next we prove a lemma which we need later in the applications.

Lemma 33. Let $A$ be a ring and $\mathfrak{p} \in \operatorname{Spec}(A)$ a prime ideal. Let $C=A_{\mathfrak{p}}, I=\mathfrak{p} C$ and $f_{1}, \ldots, f_{m} \in C_{I}^{h}$. Then there exists an étale map $A \longrightarrow B$ such that

1. $f_{1}, \ldots, f_{m} \in B$,

2. there exists a prime ideal $\mathfrak{q} \in \operatorname{Spec}(B)$ such that $\mathfrak{q} \cap A=\mathfrak{p}$.

Proof. By definition we have

$$
C_{I}^{h}=\lim _{\longrightarrow}(D \mid C \longrightarrow D \text { étale inducing } C / I=D / I D) .
$$

We choose $D$ from the inductive system above such that $f_{1}, \ldots, f_{m} \in D$. Since $(C, I)$ is a local ring and $C / I=D / I D$, the ideal $I D$ is a maximal ideal in $D$ and we have $I D \cap C=I$. Using Proposition 28 (9) for the multiplikatively closed system $S=A \backslash \mathfrak{p}$ we find an étale map $A \longrightarrow B^{\prime}$ such that $D=S^{-1} B^{\prime}$. This implies that $f_{1}, \ldots, f_{m} \in B_{g}^{\prime}$ for a suitable $g \in S$. Let $B=B_{g}^{\prime}$ and $\mathfrak{q}=I D \cap B$ then $A \longrightarrow B$ is étale having the properties 1 . and 2 .

${ }^{6}$ For the definition of excellence see 15.51 [Sta19]). 
Next we define the Henselization of an $A$-module $M$ with respect to an ideal $I \subset A$ similarly to the definition of the Henselization of $A$ with respect to $I$.

Definition 34. Let $A$ be a ring, $I \subset A$ an ideal and $M$ an $A$-module. The module

$$
M_{I}^{h}=\lim _{\longrightarrow}\left(M \otimes_{A} B \mid A \longrightarrow B \text { étale inducing } A / I=B / I B\right)
$$

is called the Henselization of $M$ with respect to $I$.

Lemma 35. $M_{I}^{h}=M \otimes_{A} A_{I}^{h}$.

Proof. The lemma follows since the direct limit commutes with the tensor product (cf. 10.75.2 [Sta19]).

Definition 36. Let $A$ be a ring, $R=A\langle x\rangle, B$ an A-algebra and $M$ an $R$-module. We define the henselian tensor product of $R$ and $B$ over $A$ as the ring

$$
\left.R \otimes_{A}^{h} B:=\lim _{\longrightarrow}(C \mid B[x] \longrightarrow C \text { étale inducing } B=C /\langle x\rangle C)=B\langle x\rangle\right) .
$$

$M \otimes_{A}^{h} B:=\lim _{\longrightarrow}\left(M \otimes_{A} C \mid B[x] \longrightarrow C\right.$ étale inducing $\left.\left.B=C /\langle x\rangle C\right)=M \otimes_{A} B\langle x\rangle\right)$.

The Henselian tensor product has similar properties as the complete tensor product. Especially we obtain the following lemma.

Lemma 37. If $A\langle x\rangle^{p} \stackrel{T}{\rightarrow} A\langle x\rangle^{q} \rightarrow M \rightarrow 0$ is an $A\langle x\rangle$-presentation of $M$ then

$$
M \otimes_{A}^{h} B=\operatorname{coker}\left(B\langle x\rangle^{p} \stackrel{T}{\rightarrow} B\langle x\rangle^{q}\right) .
$$

In particular $R \otimes_{A}^{h} k(\mathfrak{p})=k(\mathfrak{p})\langle x\rangle$ for $\mathfrak{p} \in \operatorname{Spec} A$.

Definition 38. Let $A$ be a ring, $R=A\langle x\rangle$ and $M$ an $R$-module. We define for $\mathfrak{p} \in \operatorname{Spec} A$ the $R \otimes_{A}^{h} k(\mathfrak{p})=k(\mathfrak{p})\langle x\rangle$-module

$$
M^{h}(\mathfrak{p}):=M \otimes_{A}^{h} k(\mathfrak{p})
$$

and call it the Henselian fibre of $M$ over $\mathfrak{p}$. Moreover, we set

$$
d_{\mathfrak{p}}^{h}(M):=\operatorname{dim}_{k(\mathfrak{p})} M^{h}(\mathfrak{p})
$$

\subsection{Semicontinuity for algebraically presented modules}

Let $A$ be Noetherian and $M$ finitely generated as $R=A[[x]]$-module. Then $M$ is finitely $R$-presented and in this section we assume that $M$ has an algebraic presentation matrix. That is, there exists a presentaion

$$
R^{p} \stackrel{T}{\rightarrow} R^{q} \rightarrow M \rightarrow 0
$$

with $T=\left(t_{i j}\right)$ a $q \times p$ matrix such that $t_{i j} \in A\langle x\rangle, x=\left(x_{1}, \ldots, x_{n}\right)$, the ring of algebraic power series over $A$ (cf. Definition 30), e.g. $t_{i j} \in A[x]$. Under this assumption we shall prove the semicontinuity of $\hat{d}_{\mathfrak{b}}(M)$ for $\mathfrak{b} \in \operatorname{Spec} A$.

We set $R_{0}=A\langle x\rangle$ and $M_{0}=\operatorname{coker}\left(R_{0}^{p} \stackrel{T}{\rightarrow} R_{0}^{p}\right)$. Then using the $\langle x\rangle$-adic completion we obtain $R_{0}^{\wedge}=R$ and $M_{0}^{\wedge}=M$. 
Lemma 39. Let $B \supset A$ be an A-algebra, $\mathfrak{b} \in \operatorname{Spec} B$ and $\mathfrak{a}=\mathfrak{b} \cap A$. Then

$$
\hat{d}_{\mathfrak{a}}(M)<\infty \Leftrightarrow \hat{d}_{\mathfrak{b}}\left(M \hat{\otimes}_{A} B\right)<\infty \Leftrightarrow d_{\mathfrak{b}}^{h}\left(M_{0} \otimes_{A}^{h} B\right)<\infty
$$

and

$$
\hat{d}_{\mathfrak{a}}(M)=\hat{d}_{\mathfrak{b}}\left(M \hat{\otimes}_{A} B\right)=d_{\mathfrak{b}}^{h}\left(M_{0} \otimes_{A}^{h} B\right) .
$$

Proof. $M \hat{\otimes}_{A} B$ is considered as an $R \hat{\otimes}_{A} B=B[[x]]$-module and $M_{0} \otimes_{A}^{h} B$ as $R_{0} \otimes_{A}^{h}$ $B=B\langle x\rangle$-module. Therefore we have

$$
\begin{array}{ll}
\hat{d}_{\mathfrak{a}}(M) & =\operatorname{dim}_{k(\mathfrak{a})}\left(M \hat{\otimes}_{A} k(\mathfrak{a})\right) \\
\hat{d}_{\mathfrak{b}}\left(M \hat{\otimes}_{A} B\right) & =\operatorname{dim}_{k(\mathfrak{b})}\left(M \hat{\otimes}_{A} B \hat{\otimes}_{B} k(\mathfrak{b})\right) \\
d_{\mathfrak{b}}^{h}\left(M_{0} \otimes_{A}^{h} B\right) & =\operatorname{dim}_{k(\mathfrak{b})}\left(M_{0} \otimes_{A}^{h} B \otimes_{B}^{h} k(\mathfrak{b})\right)
\end{array}
$$

and

$$
\begin{array}{ll}
M \hat{\otimes}_{A} k(\mathfrak{a}) & =\operatorname{coker}\left(k(\mathfrak{a})[[x]]^{p} \stackrel{\bar{T}}{\rightarrow} k(\mathfrak{a})[[x]]^{q}\right) \\
M \hat{\otimes}_{A} B \hat{\otimes}_{B} k(\mathfrak{b}) & =\operatorname{coker}\left(k(\mathfrak{b})[[x]]^{p} \stackrel{\bar{T}}{\rightarrow} k(\mathfrak{b})[[x]]^{q}\right) \\
M_{0} \otimes_{A}^{h} B \otimes_{B}^{h} k(\mathfrak{b}) & =\operatorname{coker}\left(k(\mathfrak{b})\langle x\rangle^{p} \stackrel{\bar{T}}{\rightarrow} k(\mathfrak{b})\langle x\rangle^{q}\right)
\end{array}
$$

with $\bar{T}=\left(\bar{t}_{i j}\right)$ and $\bar{t}_{i j}$ the induced elements in $k(\mathfrak{a})[x]$ resp. $k(\mathfrak{b})[x]$.

If $\hat{d}_{\mathfrak{b}}\left(M \hat{\otimes}_{A} B\right)<\infty$ there exists an $N_{0}$ such that $\langle x\rangle^{N} M \hat{\otimes}_{A} B \hat{\otimes}_{B} k(\mathfrak{b})=0$ for $N \geq N_{0}$ and hence

$$
\begin{aligned}
M \hat{\otimes}_{A} B \hat{\otimes}_{B} k(\mathfrak{b}) & =\operatorname{coker}(k(\mathfrak{b})[[x]])^{p} /\langle x\rangle^{N} \stackrel{\bar{T}}{\rightarrow}(k(\mathfrak{b})[[x]])^{q} /\langle x\rangle^{N} \\
& =\left(\operatorname{coker}\left(k(\mathfrak{a})[[x]] /\langle x\rangle^{N}\right)^{p} \stackrel{\bar{T}}{\rightarrow}\left(k(\mathfrak{a})[[x]] /\langle x\rangle^{N}\right)^{q}\right) \otimes_{k(\mathfrak{a})} k(\mathfrak{b}) .
\end{aligned}
$$

Since this holds for every $N \geq N_{0}$, we obtain $\hat{d}_{\mathfrak{a}}(M)<\infty$. Similarly we can see that $\hat{d}_{\mathfrak{a}}(M)<\infty$ implies $\hat{d}_{\mathfrak{b}}\left(M \otimes_{A} B\right)<\infty$ and in both cases we obtain $\hat{d}_{\mathfrak{a}}(M)=$ $\hat{d}_{\mathfrak{b}}\left(M \otimes_{A} B\right)$. This gives the first equality in the Lemma. Since $B\langle x\rangle /\langle x\rangle^{N}=$ $B[[x]] /\langle x\rangle^{N}$ we get the remaining claims.

Lemma 40. Let $(A, \mathfrak{m}, \mathbb{k})$ be a local Noetherian Henselian ring and $R$ a local quasifinite (i.e. $\operatorname{dim}_{\mathbb{k}} R / \mathfrak{m} R<\infty$ ) and finite type A-algebra in the Henselian sense ${ }^{7}$. Then $R$ is a finite A-algebra, i.e., finitely generated as an A-module.

Proof. This is an immediate consequence of Proposition 1.5 of [KPP78].

Corollary 41. Let $\left(A, \mathfrak{m}, \mathbb{k}_{\mathfrak{k}}\right)$ be a local Noetherian Henselian ring and $R$ a local and finite type A-algebra in the Henselian sense. If $M$ is a finitely generated and quasi-finite (i.e. $\left.\operatorname{dim}_{\mathbb{k}} M / \mathfrak{m} M<\infty\right) R$-module, then $M$ is a finitely generated A-module.

Proof. Passing from $R$ to $R / \operatorname{Ann}_{R}(M)$ we may assume that $\operatorname{Ann}_{R}(M)=0$. In this case $\operatorname{dim}_{\mathbb{k}} M / \mathfrak{m} M<\infty$ implies $\operatorname{dim}_{\mathbb{k}} R / \mathfrak{m} R<\infty$. Lemma 40 implies that $R$ is a finitely generated $A$-module. Since $M$ is finitely generated over $R$ it follows that $M$ is a finitely generated $A$-module.

${ }^{7} R$ is an $A$-algebra of finite type in the Henselian sense if $R=A\left\langle t_{1}, \ldots, t_{s}\right\rangle$ for suitable $t_{1}, \ldots, t_{s} \in$ $R$ 
Theorem 42. Let $A$ be a Noetherian ring, $R=A[[x]], x=\left(x_{1}, \ldots, x_{n}\right)$, and $M a$ finitely generated $R$-module admitting a presentation

$$
R^{p} \stackrel{T}{\rightarrow} R^{q} \rightarrow M \rightarrow 0
$$

with algebraic presentation matrix $T=\left(t_{i j}\right), t_{i j} \in A[x]$ or, more generally, $\in A\langle x\rangle$. Fix $\mathfrak{p} \in \operatorname{Spec} A$ with $\hat{d}_{\mathfrak{p}}(M)<\infty$. Then there is an open neighbourhood $U$ of $\mathfrak{p}$ in Spec $A$ such that

$$
\hat{d}_{\mathfrak{q}}(M) \leq \hat{d}_{\mathfrak{p}}(M) \text { for all } \mathfrak{q} \in U .
$$

Proof. Recall that $R_{0}=A\langle x\rangle$ is the Henselization of $A[x]$ with respect to $\langle x\rangle$ and $M_{0}=\operatorname{coker}\left(R_{0}^{p} \stackrel{T}{\rightarrow} R_{0}^{p}\right)$. Denote by $A^{h}$ the henselization of the local ring $A_{\mathfrak{p}}$ with respect to its maximal ideal. We set $R^{h}:=A^{h}\langle x\rangle$ and ${ }^{8} M^{h}:=\operatorname{coker}\left(\left(R^{h}\right)^{p} \stackrel{T}{\rightarrow}\right.$ $\left.\left(R^{h}\right)^{q}\right)=M_{0} \otimes^{h} R^{h}$. Then Lemma 39 implies $\hat{d}_{\mathfrak{p}}(M)=d_{\mathfrak{p}}^{h}\left(M^{h}\right)$ and Corollary 41 that $M^{h}$ is a finitely generated $A^{h}$-module $\left(R^{h}\right.$ is a finite type $A^{h}$-algebra in the Henselian sense). Lemma 33 implies that there is an étale neighbourhood $\pi:$ Spec $B \rightarrow$ Spec $A$ of $\mathfrak{p}$ such that $M_{0} \otimes_{A}^{h} B=\operatorname{coker}\left(\left(R_{0} \otimes_{A}^{h} B\right)^{p} \stackrel{T}{\rightarrow}\left(R_{0} \otimes_{A}^{h} B\right)^{q}\right)$ is a finitely generated $B$-module and $M_{0} \otimes_{A}^{h} B \otimes_{B}^{h} A^{h}=M^{h}$. Choose $\mathfrak{b} \in \operatorname{Spec} B$ such that $\mathfrak{b} \cap A=\mathfrak{p}$. This is possible because of Lemma 33. Corollary 41 and Lemma 1 imply that there is an open neigbourhood $\tilde{U} \subset \operatorname{Spec} B$ of $\mathfrak{b}$ such that for $\mathfrak{c} \in \tilde{U}$ we have $d_{\mathfrak{c}}\left(M_{0} \otimes_{A}^{h} B\right) \leq d_{\mathfrak{b}}\left(M_{0} \otimes_{A}^{h} B\right)$. Since $\pi$ is étale it is open (Proposition 28), $U:=\pi(\tilde{U})$ is an open neighbourhood of $\mathfrak{p}$ in Spec $A$ and for any $\mathfrak{q} \in U \cap \operatorname{Spec} A$ there exists a $\mathfrak{c} \in \tilde{U} \cap \operatorname{Spec} B$ with $\mathfrak{c} \cap A=\mathfrak{q}$. From Lemma 39 we obtain $\hat{d}_{\mathfrak{q}}(M)=d_{\mathfrak{c}}\left(M_{0} \otimes_{A}^{h} B\right) \leq d_{\mathfrak{b}}\left(M_{0} \otimes_{A}^{h} B\right)=\hat{d}_{\mathfrak{p}}(M)$.

The important property of Henselian local rings is that quasi-finite implies finite (in the sense of Corollary 41). Examples of Henselian local rings are quotient rings of the algebraic power series rings $A=\mathbb{k}\langle y\rangle / I$ over some field $\mathbb{k}$, and analytic $\mathbb{k}_{\text {-algebras. }}{ }^{9}$

If $A$ is a complete local ring containing a field, then any finitely generated $R$-module $M$ can be polynomially presented and semicontinuity of $\hat{d}_{\mathfrak{p}}(M)$ holds, as we show now. We start with the following proposition, based on the Weierstrass division theorem.

Proposition 43. Let $(A, \mathfrak{m}, \mathbb{k})$ be a Noetherian complete local ring containing $\mathbb{k}$, $R=A[[x]], x=\left(x_{1}, \ldots, x_{n}\right)$, and $M$ a finitely generated $R$-module such that $\operatorname{dim}_{\mathrm{k}} M / \mathfrak{m} M<\infty$. Let $J=\operatorname{Ann}_{R}(M)$. Then there exist $f_{1}, \ldots, f_{s}, s \geq n$, with the following properties:

1. $f_{i} \in A[x]$ for all $i$.

\footnotetext{
${ }^{8}$ Note that $R^{h}$ is the Henselization of $A_{\mathfrak{p}}[x]$ with respect to the maximal ideal $\langle\mathfrak{p}, x\rangle$.

${ }^{9} \mathrm{An}$ analytic $\mathbb{k}$-algebra is the quotient $\mathbb{k}\{y\} / I, y=\left(y_{1}, \ldots, y_{s}\right)$, of a convergent power series ring over a complete real-valued field $\mathbb{k}_{\mathbf{k}}(\mathrm{cf}$. [GLS07]). E.g., if $\mathbb{k}$ is any field with the trivial valuation, then $\mathbb{k}\{y\}=\mathbb{k}[[y]]$ is the formal power series ring; if $\mathbb{k} \in\{\mathbb{R}, \mathbb{C}\}$, then $\mathbb{k}\{y\}$ is the usual convergent power series ring.
} 
2. $f_{n-i+1} \in A\left[x_{1}, \ldots, x_{i}\right]$ is a Weierstrass polynomial with respect to $x_{i}$ for $i=1, \ldots, n$.

3. $J=\left\langle f_{1}, \ldots, f_{s}\right\rangle A[[x]]$.

Proof. To prove the statements we use induction on $n$, the number of the variables $x$. The assumption implies that $\operatorname{dim}_{\mathbb{k}}(R / J+\mathfrak{m} R)<\infty$, i.e. the ideal $J+\mathfrak{m} R$ is primary to the maximal ideal $\langle x\rangle+\mathfrak{m} R$ of $R$. This implies that $x_{n}^{b} \in J+\mathfrak{m} R$ for some $b$. Therefore there exists $g \in J, g=x_{n}^{b}+f$ with $f \in \mathfrak{m} R$. We know by Cohen's structure theorem that $A=\mathbb{k}[[y]] / I$ for suitable variables $y$ and an ideal $I \subset \mathbb{k}[[y]]$. We can apply in the following the Weierstrass preparation and division theorem to representatives in $\mathbb{k}[[y, x]]$ and then take residue classes mod $I$. Obviously $g$ is $x_{n}$-general. The Weierstrass preparation theorem implies $g=u h, \mathrm{u}$ a unit in $R$, and $h \in A\left[\left[x_{1}, \ldots, x_{n-1}\right]\right]\left[x_{n}\right]$ a Weierstrass polynomial with respect to $x_{n}$. To simplify the notation we assume that $g$ is already a Weierstrass polynomial with respect to $x_{n}$. Setting $R_{0}=A\left[\left[x_{1}, \ldots, x_{n-1}\right]\right]$, the Weierstrass division theorem (cf. [GLS07, Theorem I.1.8]) says that for any $f$ in $R$ there exist unique $h \in R$ and $r \in R_{0}\left[x_{n}\right]$ such that $f=h g+r, \operatorname{deg}_{x_{n}}(r) \leq b-1$. In other words, as $R_{0}$ modules we have

$$
R=R \cdot g \oplus R_{0} \cdot x_{n}^{b-1} \oplus R_{0} \cdot x_{n}^{b-2} \oplus \cdots \oplus R_{0} .
$$

We may thus assume that $J=\left\langle g_{1}, \ldots, g_{r}\right\rangle$ with $g_{1}=g$ and $g_{i} \in R_{0}\left[x_{n}\right]$ with $\operatorname{deg}_{x_{n}}\left(g_{i}\right) \leq b-1$.

If $n=1$ then $R_{0}=A$ and the claim follows from (*). If $n \geq 2$ then $M$ is a finitely generated $R_{0}$-module since

- $R /\langle g\rangle$ is finite over $R_{0}$ and

- $g \in \operatorname{Ann}_{R}(M)$, i.e. $M$ is a finitely generated $R /\langle g\rangle$-module.

Now let $J_{0}=\operatorname{Ann}_{R_{0}}(M)$. By induction hypothesis there are $f_{2}, \ldots, f_{l}, l \geq n$, such that

1. $f_{i} \in A\left[x_{1}, \ldots, x_{n-1}\right]$ for all $i$.

2. $f_{n-i+1} \in A\left[x_{1}, \ldots, x_{i}\right]$ is a Weierstrass polynomial with respect to $x_{i}$ for $i=1, \ldots, n-1$.

3. $J_{0}=\left\langle f_{2}, \ldots, f_{l}\right\rangle R_{0}$.

Now denote by $f_{1}$ be the remainder of the division of $g$ successively by $f_{2}, \ldots, f_{n}$ and by $f_{l+i}$ the remainder of $g_{i}$ by $f_{2}, \ldots, f_{n}$ for $i>1$. These are polynomials in $x_{1}, \ldots, x_{n}$. Then $f_{1}, \ldots, f_{s}$ satisfy the conditions 1 . to 3 . of the proposition.

Corollary 44. Let $(A, \mathfrak{m}, \mathbb{k})$ be a Noetherian complete local ring containing $\mathbb{k}, R=$ $A[[x]]$ and $M$ a finitely generated $R$-module such that $\operatorname{dim}_{\mathbb{k}} M / \mathfrak{m} M<\infty$. Then $M$ is polynomially presented.

Proof. Assume $M$ has a presentation matirx $T=\left(g_{i j}\right), g_{i j} \in A[[x]]$. Let $J=$ $\operatorname{Ann}_{R}(M)$. The assumption implies that $\operatorname{dim}_{\mathbb{k}} R /(J+\mathfrak{m} R)<\infty$. Using Proposition 43 we obtain that $R / J$ is a $A$-finite and $J=\left\langle f_{1}, \ldots, f_{s}\right\rangle$ with $f_{n-i+1} \in$ $A\left[x_{1}, \ldots, x_{i}\right]$ a Weierstrass polynomial with respect to $x_{i}$ for $i=1, \ldots, n, n \leq s$. This implies that $M$ has a presentation as $R / J$-module with presentation matrix 
$T$ having entries in $R / J$. Now we can divide representatives in $R$ of the entries of $T$ successively by the Weierstrass polynomials $f_{n-i+1}, i=1, \ldots, n$. The remainders are polynomials in $A[x]$ representing the entries of $T$, which proves the claim.

Let us collect the cases for which we proved that semicontinuity of $\hat{d}_{\mathfrak{p}}(M)$ holds.

Corollary 45. Let $A$ be Noetherian and $M$ a finitely generated $R$-module. Let $\mathfrak{p} \in$ Spec $A$ and assume that one of the following conditions is satisfied:

1. $M$ is finitely generated as A-module, e.g. $\operatorname{Supp}_{R}(M) \subset V(\langle x\rangle)$, or

2. $\operatorname{dim} A=1$, or

3. $M$ is algebraically $R$-presented, or

4. $(A, \mathfrak{m}, \mathbb{k})$ is a complete local ring containing a field ${ }^{10}$.

Then there is an open neighbourhood $U \subset \operatorname{Spec} A$ of $\mathfrak{p}$ such that $\hat{d}_{\mathfrak{q}}(M) \leq \hat{d}_{\mathfrak{p}}(M)$ for all $\mathfrak{q} \in U$. In particular, the quasi-completed-finite locus $\left\{\mathfrak{p} \in \operatorname{Spec} A \mid \hat{d}_{\mathfrak{p}}(M)<\right.$ $\infty\}$ is open.

Proof. Statement 1. follows from Proposition 21, statement 2. from Theorem 24 and 3. from Theorem 42. Statement 4. follows from 3. and Corollary 44.

We do not know if semicontinuity of $\hat{d}_{\mathfrak{p}}(M)$ holds in general for $A$ Noetherian of any dimension and $M$ finitely but not necessarily algebraically presented over $R$.

Remark 46. For completeness we recall cases where semicontinuity of the usual fibre dimension $d_{\mathfrak{p}}(M)$ on $\operatorname{Spec} A$ holds if $M$ is an arbitrary finitely presented $R$-module, for different (local) rings $A$ and $R$.

- $(A, \mathfrak{m}, \mathbb{k})$ local Noetherian Henselian, $R$ a finite type $A$-algebra in the Henselian sense (by Corollary 41 and Lemma 1).

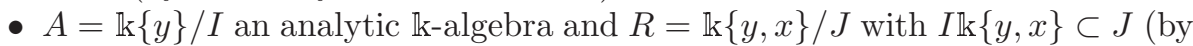
[GLS07, Theorem I.1.10]).

- $A$ a Noetherian complete local ring containing a field, $R=A[[x]]$. This is a special case of the previous item. We mention it, since $R$ is of the form considered in this paper.

- In the complex analytic situation with $A=\mathbb{C}\{y\} / I, y=\left(y_{1}, \ldots, y_{s}\right)$, and $R=$ $\mathbb{C}\{y, x\} / J, I \mathbb{C}\{y, x\} \subset J, x=\left(x_{1}, \ldots, x_{n}\right)$, we have the following stronger statement: $A \rightarrow R$ induces a morphism of complex germs $f:(X, 0) \rightarrow(Y, 0)$, $(X, 0)=V(J) \subset\left(\mathbb{C}^{n} \times \mathbb{C}^{s}, 0\right),(Y, 0)=V(I) \subset\left(\mathbb{C}^{s}, 0\right)$ and $f$ the projection. For a sufficiently small representative $f: X \rightarrow Y, M$ induces a coherent $\mathcal{O}_{X^{-}}$ module $\mathcal{F}$ on $X$ and $d_{\mathfrak{m}}(M)<\infty, \mathfrak{m}$ the maximal ideal of $A=\mathcal{O}_{Y, 0}$, means that the fibre dimension over $0 \in Y$ is finite, i.e. $d_{0}(\mathcal{F}):=\operatorname{dim}_{\mathbb{C}} \mathcal{F}_{0} / \mathfrak{m} \mathcal{F}_{0}<\infty$. Then, for sufficiently small suitable $X$ and $Y$, is $f \mid \operatorname{Supp} \mathcal{F}$ is a finite morphism

${ }^{10} \mathrm{By}$ Cohen's structure theorem this is equivalent to $A \cong \mathbb{k}[[y]] / I, \mathbb{k}$ a field. 
and $f_{*} \mathcal{F}$ is a coherent $\mathcal{O}_{Y}$-module (cf. [GLS07, Theorem I.1.67]). It follows that

$$
d_{y}(\mathcal{F}):=\operatorname{dim}_{\mathbb{C}} f_{*} \mathcal{F} \otimes_{\mathcal{O}_{Y, y}} \mathbb{C}=\sum_{z \in f^{-1}(y)} \operatorname{dim}_{\mathbb{C}} \mathcal{F}_{z} / \mathfrak{m}_{y} \mathcal{F}_{z}
$$

is upper semicontinuous at $0 \in Y$, i.e. 0 has an open neighbourhood $U \subset Y$ such that $d_{y}(\mathcal{F}) \leq d_{0}(\mathcal{F})$ for all $y \in U$.

In the above cases $M$ is finite over $A$ if it is quasi-finite over $A$ and hence semicontinuity of $d_{\mathfrak{p}}(M)$ holds by Lemma 1 . Example 19 shows that for $A$ an affine ring and $R=A[[x]]$ semicontinuity of $d_{\mathfrak{p}}(M)$ does in general not even hold for polynomially presented modules.

\subsection{Related results}

Instead of families of power series let us now consider families of algebras of finite type, a situation which is quite common in algebraic geometry. We treat the more general case of families of modules.

Let $A$ be a ring, $R=A[x] / I$ of finite type over $A$ and $M$ a finitely presented $R$-module. $M$ is called quasi-finite at $\mathfrak{n} \in \operatorname{Spec} R$ over $A$ if $\operatorname{dim}_{k(\mathfrak{p})} M_{\mathfrak{n}} / \mathfrak{p} M_{\mathfrak{n}}<\infty$ with $\mathfrak{p} \in \operatorname{Spec} A$ lying under $\mathfrak{n}$. $M$ is called quasi-finite over $\mathfrak{p} \in \operatorname{Spec} A$ if it is quasi-finite at all primes $\mathfrak{n} \in \operatorname{Spec} R$ lying over $\mathfrak{p}$, and $M$ is quasi-finite over $A$ if it is quasi-finite at all primes $\mathfrak{n} \in \operatorname{Spec} R$. The following proposition is a generalization of results from [Sta19], where the case of ring maps is treated.

Proposition 47. Let $A$ be a ring, $R$ an A-algebra of finite type over $A, M$ a finitely presented $R$-module and $f: \operatorname{Spec} R \rightarrow \operatorname{Spec} A$ the induced map of schemes.

1. The following are equivalent:

(a) $M$ is quasi-finite over $A$,

(b) $d_{\mathfrak{p}}(M)=\operatorname{dim}_{k(\mathfrak{p})} M(\mathfrak{p})=\sum_{\mathfrak{n} \in f^{-1}(\mathfrak{p})} \operatorname{dim}_{k(\mathfrak{p})} M_{\mathfrak{n}} / \mathfrak{p} M_{\mathfrak{n}}<\infty \forall \mathfrak{p} \in \operatorname{Spec} A$,

(c) The induced map $A \rightarrow S:=R / \operatorname{Ann}_{R}(M)$ is quasi-finite.

2. (Zariski's main theorem for modules). The quasi-finite locus of $M$

$$
\{\mathfrak{n} \in \operatorname{Spec} R \mid M \text { is quasi-finite at } \mathfrak{n}\}
$$

is open in $\operatorname{Spec} R$.

Proof. 1. $(a) \Rightarrow(b)$ : We have to show that the support of $M(\mathfrak{p})$ is finite. By [Sta19, Lemma 29.19.10], if $R=A[x] / I$ is a ring of finite type and quasi-finite over $A$, the induced map $f: \operatorname{Spec} R \rightarrow \operatorname{Spec} A$ has finite fibres $R(\mathfrak{p})=R \otimes_{A} k(\mathfrak{p})=$ $k(\mathfrak{p})[x] / I(\mathfrak{p})$. It follows that 2 . holds if $M$ is a ring of finite type over $A$.

In the general case let $I=\operatorname{Ann}_{R}(M)$. Then $S=R / I$ is of finite type over $A$, $M$ is finitely presented over $S$ and hence $\operatorname{Supp}(S)=\operatorname{Supp}(M)$. Moreover, let $J(\mathfrak{p})$ be the annihilator of the finitely generated $R(\mathfrak{p})$-module $M(\mathfrak{p})$ satisfying $V(J(\mathfrak{p}))=$ $\operatorname{Supp}(M(\mathfrak{p}))$. Since $R(\mathfrak{p})$ is Noetherian and $\operatorname{dim}_{k(\mathfrak{p})} M_{\mathfrak{n}}(\mathfrak{p})<\infty$ by assumption, we have $\mathfrak{n}^{N} M_{\mathfrak{n}}(\mathfrak{p})=0$ for some $N$ by Nakayama's lemma. Hence $\mathfrak{n}^{N} \subset J(\mathfrak{p}) R_{\mathfrak{n}}(\mathfrak{p})$ and $\operatorname{dim}_{k(\mathfrak{p})} R_{\mathfrak{n}}(\mathfrak{p}) / J(\mathfrak{p}) R_{\mathfrak{n}}(\mathfrak{p})<\infty$. In general, the annihilator is not compatible with 
base change, hence $I(\mathfrak{p})$ is in general different from $J(\mathfrak{p})$. But for a finitely presented module the annihilator coincides up to radical with a Fitting ideal, and Fitting ideals are compatible with base change. It follows that $\sqrt{J(\mathfrak{p}) R_{\mathfrak{n}}(\mathfrak{p})}=\sqrt{I(\mathfrak{p}) R_{\mathfrak{n}}(\mathfrak{p})}$ and therefore $\operatorname{dim}_{k(\mathfrak{p})} R_{\mathfrak{n}}(\mathfrak{p}) / I(\mathfrak{p}) R_{\mathfrak{n}}(\mathfrak{p})=\operatorname{dim}_{k(\mathfrak{p})} S_{\mathfrak{n}}(\mathfrak{p})<\infty$, which means that $A \rightarrow S$ is quasi-finite at $\mathfrak{n}$ ([Sta19, Definition 10.121.3]). Since this holds for each $\mathfrak{n} \in \operatorname{Spec} R$, the map $A \rightarrow S$ is quasi-finite and by [Sta19, Lemma 29.19.10] the set $\operatorname{Supp}(M(\mathfrak{p}))=\operatorname{Supp}(S(\mathfrak{p}))$ is finite.

$(b) \Rightarrow(c)$ : If $d_{\mathfrak{p}}(M)<\infty$ for all $\mathfrak{p} \in \operatorname{Spec} A$, then $\operatorname{dim}_{k(\mathfrak{p})} M_{\mathfrak{n}} / \mathfrak{p} M_{\mathfrak{n}}<\infty$ for all $\mathfrak{n}$ and $\mathfrak{p}$ under $\mathfrak{n}$. Then $A \rightarrow S$ is quasi-finite by the previous step.

$(c) \Rightarrow(a)$ : If $A \rightarrow S$ is quasi-finite then $\operatorname{dim}_{k(\mathfrak{p})} S_{\mathfrak{n}}(\mathfrak{p})<\infty$ for all $\mathfrak{p}$ and $\mathfrak{n}$ over $\mathfrak{p}$. Since $M_{\mathfrak{n}}(\mathfrak{p})$ is finitely presented as $S_{\mathfrak{n}}(\mathfrak{p})$-module, $\operatorname{dim}_{k(\mathfrak{p})} M_{\mathfrak{n}}(\mathfrak{p})<\infty$ for all $\mathfrak{p}$ and $\mathfrak{n}$ over $\mathfrak{p}$ and $M$ is quasi-finite over $A$.

2. In the first and third step of 1 . we proved $\operatorname{dim}_{k(\mathfrak{p})} S_{\mathfrak{n}}(\mathfrak{p})<\infty$ if and only if $\operatorname{dim}_{k(\mathfrak{p})} M_{\mathfrak{n}}(\mathfrak{p})<\infty$, and hence $M$ is quasi-finite at $\mathfrak{n}$ iff $A \rightarrow S$ is quasi-finite at $\mathfrak{n}$. It follows from a version of Zarisk's main theorem as proved in [Sta19, Lemma 10.122.13] that the set $\{\mathfrak{n} \in \operatorname{Spec} S \mid A \rightarrow S$ is quasi-finite at $\mathfrak{n}\}$ is open in Spec $S$ and thus of the form $U \cap S$ with $U$ open in $\operatorname{Spec} R$. If $\mathfrak{n} \in V=\operatorname{Spec} R \backslash \operatorname{Spec} S$ then $M_{\mathfrak{n}}(\mathfrak{p})=0$, hence $M$ is quasi-finite at $\mathfrak{n} \in V$. Thus, the quasi-finite locus of $M$ is the open set $U \cup V$.

Example 48. In the situation of Proposition 47, although the quasi-finite locus of $M$ is open in $\operatorname{Spec} R$, we cannot expect semicontinuity of $d_{\mathfrak{p}}(M)$ on $\operatorname{Spec} A$. We give an example showing that the vanishing locus of $d_{\mathfrak{p}}(M)$ is not open in Spec $A$ : Let $K$ be an algebraically closed field, $A=K[y], R=A[x]$ and $M=R /\langle x y-1\rangle$. Then $M$ is quasi-finite over $A$ but $d_{\mathfrak{p}}(M)$ is not semicontinuous since $d_{\mathfrak{p}}(M)=0$ if $\mathfrak{p}=\langle y\rangle$ and $d_{\mathfrak{p}}(M)=1$ otherwise.

By Corollary 45.4, semicontinuity of $\hat{d}_{\mathfrak{p}}(M)$ holds for $M$ a finitely generated $A[[x]]$-module if $(A, \mathfrak{m})$ is a complete Noetherian local ring containing a field under the assumption that $\operatorname{Supp}_{A}(M)=A$ but $\operatorname{Supp}_{R}(M) \not \subset V(\langle x\rangle)$ (the difficult case). The question arose whether completeness was necessary. The following example shows that this is not the case.

Example 49. We give an example of a non-complete local ring $(A, \mathfrak{m})$ and a finitely presented $R=A[[x]]$-module $M$ which is also a finitely presented $A$-module with $\operatorname{Supp}_{R}(M)$ being not contained in $V(\langle x\rangle)$ and $\operatorname{Ann}_{A}(M)=0$.

Let $\mathbb{k}$ be a field and $t_{1}, t_{2}$ independent variables. Let $A=\mathbb{k}\left[t_{1}\right]\left\langle t_{1}\right\rangle\left[\left[t_{2}\right]\right]$ and $R=A[[x]]$ with $\mathrm{x}$ a single variable. The $\operatorname{ring} A$ is local with maximal ideal $\mathfrak{m}=\left\langle t_{1}, t_{2}\right\rangle A$ and not complete. Let $M=R /\left\langle x-t_{2}\right\rangle$. Then $\operatorname{Ann}_{R}(M)=\left\langle x-t_{2}\right\rangle$ and $\operatorname{Supp}_{R}(M)=V\left(\left\langle x-t_{2}\right\rangle \not \subset V(\langle x\rangle)\right.$ and $\operatorname{Ann}_{A}(M)=\left\langle x-t_{2}\right\rangle \cap A=(0)$. Since $M$ is polynomially presented, $\hat{d}_{\mathfrak{p}}(M)$ is semicontinuous.

Let $M$ be a finitely presented $R=A[[x]]$-module and also finitely presented as an $A$-module with $A$ Noetherian. In Proposition 21 we have shown the semicontinuity of $d_{\mathfrak{p}}(M)$ and $\hat{d}_{\mathfrak{p}}(M)$ on Spec $A$, as well as the inequality $\hat{d}_{\mathfrak{p}}(M) \leq d_{\mathfrak{p}}(M)$. The following example shows that $\hat{d}_{\mathfrak{p}}(M)<d_{\mathfrak{p}}(M)$ may happen. 
Example 50. A modification of Example 19 shows that $\hat{d}_{\mathfrak{p}}(M) \neq d_{\mathfrak{p}}(M)$ may happen for $A$ a power series ring. Let $A=K[[t]], K$ a field, $R=A[[x]]$, and let $M=R /\langle t-x\rangle \cong K[[t]]$. For the two prime ideals $\langle 0\rangle$ and $\langle t\rangle$ of $A$ we get:

$k(\langle 0\rangle)=K((t)), k(\langle t\rangle)=K, M(\langle 0\rangle) \cong K[[t]] \otimes_{K[t t]]} K((t))=K((t))$, $M(\langle t\rangle) \cong K$ and $d_{\langle 0\rangle}(M)=d_{\langle t\rangle}(M)=1$. Hence $d_{\mathfrak{p}}(M)$ is semicontinuous (even continuous) on Spec $A$ as predicted in Remark 46, third item.

$\hat{M}(\langle 0\rangle) \cong K((t))[[x]] /\langle t-x\rangle=0, \hat{M}(\langle t\rangle) \cong K$ and $\hat{d}_{\langle 0\rangle}(M)=0, \hat{d}_{\langle t\rangle}(M)=1$. Hence $d_{\mathfrak{p}}(M)$ is semicontinuous on Spec $A$ as predicted by Corollary 45 . Note that $M$ is finitely presented as $A$-module and we have $\hat{d}_{\langle 0\rangle}(M)<d_{\langle 0\rangle}(M)$.

\section{Singularity invariants}

\subsection{Isolated singularities and flatness}

Recall that a local Noetherian ring $(A, \mathfrak{m})$ is said to be regular if $\mathfrak{m}$ can be generated by $\operatorname{dim} A$ elements. A Noetherian $\operatorname{ring} A$ is said to be regular if the local ring $A_{\mathfrak{p}}$ is regular for all $\mathfrak{p} \in \operatorname{Spec} A$. For arbitrary Noetherian rings the regular locus $\operatorname{Reg} A:=\left\{\mathfrak{p} \in \operatorname{Spec} A \mid A_{\mathfrak{p}}\right.$ is regular $\}$ need not be open in Spec $A$. However, $\operatorname{Reg} A$ is open if $A$ is a complete Noetherian local ring ([Mat86, Corollary of Theorem $30.10])$ and the non-regular locus $\left\{\mathfrak{p} \in \operatorname{Spec} A \mid A_{\mathfrak{p}}\right.$ is not regular $\}$ is closed.

However, in our situation of families of power series, the notion of formal smoothness is more appropriate than that of regularity. Formal smoothness is a relative notion and refers to a morphism, while regularity is an absolute property of the ring. The notions are related as follows. Let $(A, \mathfrak{m})$ be a local ring containing a field $\mathbb{k}$. If $A$ is formally smooth over $\mathbb{k}$ (w.r.t. the $\mathfrak{m}$-adic topology) then $A$ is regular and the converse holds if the residue field $A / \mathfrak{m}$ is separable over $\mathbb{k}$ (see Remark 52). Hence formal smoothness of $A$ over $\mathbb{k}$ coincides with regularity if $\mathbb{k}$ is a perfect field. The notions also coincide for arbitrary $\mathbb{k}$ if $A$ is the quotient ring of a formal power series ring over $\mathbb{k}$ by an ideal (cf. Lemma 57 ).

We recall now basic facts about formal smoothness. For details and proofs see [Mat86] and [Maj10].

Definition 51. Let $A$ be a ring, $B$ an A-algebra defined by $\phi: A \longrightarrow B$ and $I$ an ideal in $B$. The $A$-algebra $B$ is called formally smooth with respect to the $I$ adic topology (for short $B$ is $I$-smooth over $A$ ) if for any $A$-algebra $C$ and any continuous $^{11} A$-algebra homomorphism $u: B \longrightarrow C / N, N$ an ideal in $C$ with $N^{2}=0$, there exist $\sigma: B \longrightarrow C$ such that $\pi \sigma=u$.

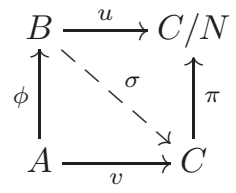

11 Here we consider $B$ with the $I$-adic topology and $C / N$ with the discrete topology; $u$ is continuous if $u\left(I^{m}\right)=0$ for some $m$. 
If $I=0$ then $B$ is called $a$ formally smooth $A$-algebra.

Remark 52. 1. A formally smooth map of finite presentation is smooth ([Sta19] Proposition 10.137.13).

2. $A[x], x=\left(x_{1}, \ldots, x_{n}\right)$, is smooth over $A$ ([Sta19] Lemma 10.137.4).

3. $A[[x]]$ is $\langle x\rangle$-smooth over $A$ ([Mat86] page 215 ).

4. Let $(A, \mathfrak{m})$ be a local ring containing a field $\mathbb{k}$.

(a) $A$ is $\mathfrak{m}$-smooth over $\mathbb{k}$ iff $A$ is geometrically regular, i.e. $A \otimes_{\mathbb{k}} \mathbb{k}^{\prime}$ is a regular ring for every finite extension field $\mathbb{k}^{\prime}$ of $\mathbb{k}$ ([Mat86, Theorem 28.7 ).

(b) Assume that $A / \mathfrak{m}$ is separable over $\mathbb{k}$. Then $A$ is $\mathfrak{m}$-smooth over $\mathbb{k}$ iff $A$ is regular ([Mat86] Lemma 1, page 216).

We now generalize example 1 on page 215 of [Mat86].

Lemma 53. Let $A$ be a ring, $B$ a A-algebra, $I$ an ideal in $B$ and $\widehat{B}$ the $I$-adic completion of $B . \phi: A \longrightarrow B$ is I-smooth iff $\hat{\phi}: A \longrightarrow \widehat{B}$ is I $\widehat{B}$-smooth.

Proof. Assume that $B$ is $I$-smooth over $A$ and consider the following commutative diagram:

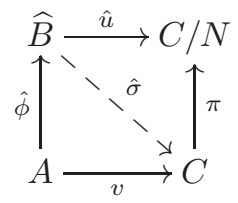

with $N^{2}=0$. We have to prove that there exists $\hat{\sigma}$ such that $\pi \hat{\sigma}=\hat{u}$. Since $\hat{u}$ is continuous there exist $m$ such that $\hat{u}\left(I^{n} \widehat{B}\right)=0$. Let $i: B \longrightarrow \widehat{B}$ be the cononical map such that $\hat{\phi}=i \phi$. The $I$-smoothness of $B$ implies that there exists $\sigma: B \longrightarrow C$ such that $\sigma \pi=\hat{u} i . \hat{u}\left(I^{n} \widehat{B}\right)=0$ implies $\sigma\left(I^{m}\right) \subset N$. Since $N^{2}=0$ we obtain $\sigma\left(I^{2 m}\right)=0$. We obtain the following commutative diagram:

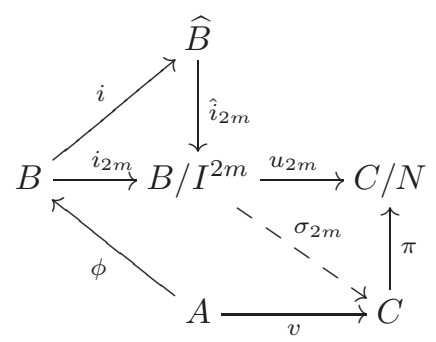

Now we define $\hat{\sigma}=\sigma_{2 m} \hat{i}_{2 m}$. This proves that $\hat{\phi}: A \longrightarrow \widehat{B}$ is $I \widehat{B}$-smooth. 
Now assume that $\hat{\phi}: A \longrightarrow \widehat{B}$ is $I \widehat{B}$-smooth. Consider the following commutative diagram:

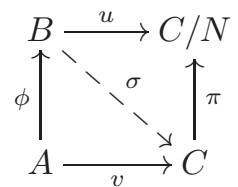

with $N^{2}=0$. We have to prove that there exists $\sigma$ such that $\pi \sigma=u$. Since $\hat{\phi}: A \longrightarrow \widehat{B}$ is $I \widehat{B}$-smooth there exists $\hat{\sigma}: \widehat{B} \longrightarrow C$ with $\pi \hat{\sigma}=\hat{u}$. Now we define $\sigma=\hat{\sigma} i$ and obtain $\pi \sigma=u$.

The following important theorem is due to Grothendieck ([Mat86] Theorem 28.9).

Theorem 54. Let $(A, \mathfrak{m})$ be a local ring and $(B, \mathfrak{n})$ a local $A$-algebra. Let $\bar{B}=B / \mathfrak{m} B$ and $\overline{\mathfrak{n}}=\mathfrak{n} / \mathfrak{m} B$. Then $B$ is $\mathfrak{n}$-smooth over $A$ iff $\bar{B}$ is $\overline{\mathfrak{n}}$-smooth over $A / \mathfrak{m}$ and $B$ is flat over $A$.

Definition 55. Let $A$ be $a$ ring and $B$ an $A$-algebra defined by $\phi: A \longrightarrow B$. We define the smooth locus of $\phi$ by

$$
\operatorname{Sm}(\phi):=\left\{P \in \operatorname{Spec}(B) \mid A_{\phi^{-1}(P)} \longrightarrow B_{P} \text { is } P \text {-smooth }\right\} .
$$

and the singular locus of $\phi$ by

$$
\operatorname{Sing}(\phi):=\operatorname{Spec}(B) \backslash \operatorname{Sm}(\phi) .
$$

Remark 56. Let $A$ be a ring and $B$ an $A$-algebra defined by $\phi: A \longrightarrow B$. The Theorem 54 of Grothendieck implies that

$$
\begin{aligned}
\operatorname{Sm}(\phi)= & \left\{P \in \operatorname{Spec}(B) \mid A_{Q} \longrightarrow B_{P}, Q=\phi^{-1}(P),\right. \text { is flat } \\
& \text { and } \left.B_{P} / Q B_{P} \text { is } P B_{P} / Q B_{P}-\text { smooth over } k(Q)\right\} .
\end{aligned}
$$

Now let $\mathbb{k}$ be a field, $\mathbb{k}[[x]], x=\left(x_{1}, \cdots, x_{n}\right)$, the formal power series ring over $\mathbb{k}$ and $I$ an ideal in $\langle x\rangle \mathbb{k}[[x]]$. If $I$ is generated by $f_{1}, \ldots, f_{m}$ we denote by $J a c(I)$ the Jacobian matrix $\left(\partial f_{j} / \partial x_{i}\right)$ and by $I_{k}(J a c(I))$ the ideal generated by the $k \times k$ minors of $\operatorname{Jac}(I)$ (which is independent of the chosen generators $f_{j}$ ). The following lemma gives equivalent conditions for the maximal ideal $\langle x\rangle \in B=\mathbb{k}[[x]] / I$ to be contained in the smooth locus $\operatorname{Sm}(\phi)$ of the map $\phi: \mathbb{k} \rightarrow B(\operatorname{Remark} 56)$.

Lemma 57. If $\operatorname{dim} \mathbb{k}[[x]] / I=d$ the following are equivalent.

1. $\mathbb{k}[[x]] / I$ is $\langle x\rangle$-smooth over $\mathbb{k}$.

2. $\mathbb{k}[[x]] / I$ is regular.

3. $I_{d}(\operatorname{Jac}(I))=\mathbb{k}[[x]]$ (Jacobian criterion).

4. $\mathbb{k}[[x]] / I \cong \mathbb{k}\left[\left[y_{1}, \ldots, y_{d}\right]\right]$.

Proof. The equivalence of 1 . and 2. follows from [Mat86, Lemma 1, p. 216], the equivalence of 3 . and 4 . is the inverse mapping theorem for formal power series ${ }^{12}$.

${ }^{12}$ Given $f_{1}, \ldots, f_{n} \in \mathbb{k}\left[\left[x_{1}, \ldots, x_{n}\right]\right]$ then $\operatorname{det}\left(\frac{\partial f_{i}}{\partial x_{j}}\right)$ is a unit iff $\mathbb{k}\left[\left[x_{1}, \ldots, x_{n}\right]\right]=\mathbb{k}\left[\left[f_{1}, \ldots, f_{n}\right]\right]$ ([GLS07, Theorem I.1.18]). 
Obviously 4. implies 2. From [Mat86, Theorem 29.7, p. 228 in] we deduce that 2. implies 4.

Remark 58. Part of the lemma can be generalized by extending the proof of Theorem 30.3 in [Mat86] as follows:

Let $P$ be a prime ideal in $\mathbb{k}[[x]]$ containing $I=\left\langle f_{1}, \ldots, f_{m}\right\rangle$ and $\mathfrak{m}$ the maximal ideal of $A=\mathbb{k}[[x]]_{P} / I \mathbb{k}[[x]]_{P}$. Then $I_{d}(\operatorname{Jac}(I))=\mathbb{k}[[x]]_{P}$ implies that $A$ is $\mathfrak{m}$-smooth over $\mathbb{k}$ (or geometric regular by Remark 52 ).

We use the Jacobian criterion to define the singular locus of ideals in power series rings over a field.

Definition 59. 1. If $B=\mathbb{k}[[x]] / I$ is pure $d$-dimensional (i.e. $\operatorname{dim} B / P=d$ for all minimal primes $P \in \operatorname{Spec} B$ ) we define the singular locus of $B$ (or of $I$ ) as

$$
\operatorname{Sing}(B)=V\left(I+I_{d}(J a c(I)) .\right.
$$

2. If $B$ is not pure dimensional we consider the minimal primes $P_{1}, \ldots, P_{r}$ of $B$. Then $B / P_{i}$ is pure dimensional and we define the singular locus of $B$ as

$$
\operatorname{Sing}(B)=\bigcup_{i=1}^{r} \operatorname{Sing}\left(B / P_{i}\right) \cup \bigcup_{i \neq j} V\left(P_{i}\right) \cap V\left(P_{j}\right),
$$

which is a closed subscheme of $\operatorname{Spec} B$. The points in $\operatorname{Spec} B \backslash \operatorname{Sing}(B)$ are called non-singular points of $B$.

3. We say that $\mathbb{k}[[x]] / I$ (or I) has an isolated singularity (at 0 ) if the maximal ideal $\langle x\rangle$ is an isolated point of $\operatorname{Sing}(\mathbb{k}[[x]] / I)$ or if $\langle x\rangle$ is a non-singular point.

Remark 60. Let $i: \mathbb{k} \longrightarrow B$ be the obvious inclusion. Then $\operatorname{Sing}(B)=\operatorname{Sing}(i)$ whenever $\mathbb{k}$ is perfect, but not in general. A counterexample is given for instance, by letting $\operatorname{char}(\mathbb{k})=p>0, a \in \mathbb{k} \backslash \mathbb{k}^{p}$ and $B=\mathbb{k}\left[\left[x_{1}, x_{2}\right]\right] /\left\langle x_{1}^{p}-a x_{2}^{p}\right\rangle$.

Note that $\operatorname{Sing}(B)$ carries a natural scheme structure given by the Fitting ideal $I+I_{d}(J a c(I)) \subset \mathbb{k}[[x]]$ if $B$ is pure $d$-dimensional. In general we endow $\operatorname{Sing}(B)$ with its reduced structure.

Now let us consider families. Let $A$ be a Noetherian $\operatorname{ring}, F_{1}, \ldots, F_{m} \in$ $\langle x\rangle A[[x]], I \subset A[[x]]$ the ideal generated by $F_{1}, \ldots, F_{m}$ and set $B:=A[[x]] / I$. We describe now the smooth locus of the map $\phi: A \rightarrow B$ along the section $\sigma: \operatorname{Spec} A \rightarrow \operatorname{Spec} B, \mathfrak{p} \mapsto \mathfrak{n}_{\mathfrak{p}}=\langle x, \mathfrak{p}\rangle$, of $\operatorname{Spec} \phi$.

For $\mathfrak{p} \in \operatorname{Spec} A$ denote by $F_{i}(\mathfrak{p})$ the image of $F_{i}$ in $k(\mathfrak{p})[[x]]$. Note that $F_{1}(\mathfrak{p}), \ldots, F_{m}(\mathfrak{p})$ generate the ideal $\hat{I}(\mathfrak{p}) \subset k(\mathfrak{p})[[x]]$, and that we have (by Lemma 15.3) for the completed fibre of $\phi$ over $\mathfrak{p}$

$$
\hat{B}(\mathfrak{p})=\left(B_{\mathfrak{n}_{\mathfrak{p}}} / \mathfrak{p} B_{\mathfrak{n}_{\mathfrak{p}}}\right)^{\wedge}=k(\mathfrak{p})[[x]] / \hat{I}(\mathfrak{p}) .
$$

The maximal ideals of the local rings of the fibre $B_{\mathfrak{n}_{\mathfrak{p}}} / \mathfrak{p} B_{\mathfrak{n}_{\mathfrak{p}}}$ and the completed fibre $\hat{B}(\mathfrak{p})$ are generated by $\mathfrak{n}_{\mathfrak{p}} / \mathfrak{p}=\langle x\rangle$. Assume that $\phi: A \rightarrow B$ is flat. Then the 
theorem of Grothendieck says

$$
\begin{aligned}
\mathfrak{n}_{\mathfrak{p}} \in \operatorname{Sm}(\phi) & \Leftrightarrow B_{\mathfrak{n}_{\mathfrak{p}}} \text { is } \mathfrak{n}_{\mathfrak{p}} \text {-smooth over } A_{\mathfrak{p}} \\
& \Leftrightarrow B_{\mathfrak{n}_{\mathfrak{p}}} / \mathfrak{p} B_{\mathfrak{n}_{\mathfrak{p}}} \text { is }\langle x\rangle \text {-smooth over } k(\mathfrak{p}) .
\end{aligned}
$$

Lemma 61. With the above notations assume that $\phi: A \rightarrow B$ is flat. Denote by

$$
\operatorname{Sing}_{\sigma}(\phi):=\left\{\mathfrak{n}_{\mathfrak{p}} \in \operatorname{Spec} B \mid B_{\mathfrak{n}_{\mathfrak{p}}} \text { is not } \mathfrak{n}_{\mathfrak{p}} \text {-smooth over } A_{\mathfrak{p}}\right\}
$$

the singular locus of $\phi$ along the section $\sigma$. Then

$$
\operatorname{Sing}_{\sigma}(\phi)=\left\{\mathfrak{n}_{\mathfrak{p}} \in \operatorname{Spec} B \mid \hat{B}(\mathfrak{p}) \text { is not regular }\right\} \text {. }
$$

Proof. $B_{\mathfrak{n}_{\mathfrak{p}}} / \mathfrak{p} B_{\mathfrak{n}_{\mathfrak{p}}}$ is $\langle x\rangle$-smooth over $k(\mathfrak{p})$ iff $\left(B_{\mathfrak{n}_{\mathfrak{p}}} / \mathfrak{p} B_{\mathfrak{n}_{\mathfrak{p}}}\right)^{\wedge}=k(\mathfrak{p})[[x]] / \hat{I}(\mathfrak{p})$ is $\langle x\rangle$ smooth over $k(\mathfrak{p})$ by Lemma 53. The claim follows from Lemma 57.

Since we assumed $B$ to be flat over $A$, we have $\operatorname{dim} \hat{B}(\mathfrak{p})=\operatorname{dim} B_{\mathfrak{n}_{\mathfrak{p}}}-\operatorname{dim} A_{\mathfrak{p}}$ (by [Mat86, Theorem 15.1]). If $\phi$ is of pure relative dimension $d$ (i.e. $\hat{B}(\mathfrak{p})$ is pure $d$-dimensional for all $\mathfrak{p}$ ) then Lemma 57 implies

$$
\operatorname{Sing}_{\sigma}(\phi)=\left\{\mathfrak{n}_{\mathfrak{p}} \in \operatorname{Spec} B \mid \hat{I}_{d}(\operatorname{Jac}(I))(\mathfrak{p}) \text { is a proper ideal of } k(\mathfrak{p})[[x]]\right\},
$$

where $\operatorname{Jac}(I)$ is the Jacobian matrix $\left(\partial F_{j} / \partial x_{i}\right)$ and $I_{d}(J a c(I)) \subset A[[x]]$ the ideal defined by the $d \times d$-minors.

\subsection{Milnor number and Tjurina number of hypersurface singularities}

Let $\mathbb{k}$ be a field and $f \in \mathbb{k}[[x]], x=\left(x_{1}, \cdots, x_{n}\right)$ a formal power series. The most important invaraints are the Milnor number $\mu(f)$ and the Tjurina number $\tau(f)$, defined as

$$
\begin{aligned}
\mu(f) & =\operatorname{dim}_{\mathbb{k}} \mathbb{k}[[x]] / j(f), \\
\tau(f) & =\operatorname{dim}_{\mathbb{k}_{k}} \mathbb{k}[[x]] /\langle f, j(f)\rangle,
\end{aligned}
$$

where $j(f)=\left\langle\partial f / \partial x_{1}, \ldots, \partial f / \partial x_{n}\right\rangle$ is the Jacobian ideal of $f$. We say that $f$ has an isolated critical point (at 0) resp. an isolated singularity (at 0) if $\mu(f)<\infty$ resp. $\tau(f)<\infty$. Note that $\tau(f)<\infty$ iff $\mathbb{k}[[x]] /\langle f\rangle$ has an isolated singularity in the sense of Definition 59.

Remark 62. Let $\operatorname{char}(\mathbb{k})=0$. It is proved in [BGM12, Theorem 2] that for $f \in\langle x\rangle$, $\mu(f)<\infty \Leftrightarrow \tau(f)<\infty$ but it is easy to see that this is not true in positive characteristic. We have always $\tau(f) \leq \mu(f)$ and $\tau(f)=\mu(f) \Leftrightarrow f \in j(f)$. If $\mathbb{k}=\mathbb{C}$ and if $f \in\langle x\rangle^{2}$ has an isolated singularity, this is equivalent to $f$ being quasi homogeneous by a theorem of K. Saito (see [Sa71]). His proof generalises to any algebraically closed field of characteristic zero (cf. [BGM11, Theorem 2.1]).

We consider now families of singularities. Let $A$ be a Noetherian ring and $F \in R=A[[x]]$. Set

$$
j(F):=\left\langle\partial F / \partial x_{1}, \ldots, \partial F / \partial x_{n}\right\rangle
$$

and for $\mathfrak{p} \in \operatorname{Spec} A$ denote by $F(\mathfrak{p})$ the image of $F$ in $k(\mathfrak{p})[[x]]$. Then the Milnor number

$$
\mu(F(\mathfrak{p}))=\operatorname{dim}_{k(\mathfrak{p})} k(\mathfrak{p})[[x]] / j(F(\mathfrak{p}))
$$


and the Tjurina number

$$
\tau(F(\mathfrak{p}))=\operatorname{dim}_{k(\mathfrak{p})} k(\mathfrak{p})[[x]] /\langle F(\mathfrak{p}), j(F(\mathfrak{p}))\rangle
$$

are defined, and we deduce now the semicontinuity of $\mu(F(\mathfrak{p}))$ and $\tau(F(\mathfrak{p}))$.

Proposition 63. Let $A$ be Noetherian, $F \in R=A[[x]]$ and $\mathfrak{p} \in \operatorname{Spec} A$. Assume that $V(j(F)) \subset V(\langle x\rangle)$ resp. $V(\langle F, j(F)\rangle) \subset V(\langle x\rangle)$ (as sets), or $\operatorname{dim} A=1$, or $F \in A[x]$, or $A$ is a complete local ring containing a field. Then $\mu(F(\mathfrak{p}))$ and $\tau(F(\mathfrak{p}))$ are semicontinuous at $\mathfrak{p} \in \operatorname{Spec} A$.

Proof. Set $M=R / j(F)$ resp. $M=R /\langle F, j(F)\rangle$, then $\operatorname{Supp}_{R}(M)=V(j(F))$ resp. $\operatorname{Supp}_{R}(M)=V(\langle F, j(F)\rangle)$. Using Lemma 15 we get $\hat{d}_{\mathfrak{q}}(M)=\mu(F(\mathfrak{q}))$ resp. $\hat{d}_{\mathfrak{q}}(M)=\tau(F(\mathfrak{q}))$ for $\mathfrak{q} \in \operatorname{Spec} A$. The result follows from Corollary 45 .

Corollary 64. Let $F \in \mathbb{Z}[x], p \in \mathbb{Z}$ a prime number and denote by $F_{p}$ the image of $F$ in $\mathbb{F}_{p}[[x]]$ and by $F_{0}$ the image of $F$ in $\mathbb{Q}[[x]]$.

If $\mu\left(F_{p}\right)$ is finite, then $\mu\left(F_{p}\right) \geq \mu\left(F_{0}\right)$ and $\mu\left(F_{p}\right) \geq \mu\left(F_{q}\right)$ for all except finitely many prime numbers $q \in \mathbb{Z}$. In particular, if $\mu\left(F_{p}\right)$ is finite for some $p$ then $\mu\left(F_{0}\right)$ is finite.

If $\mu\left(F_{0}\right)$ is finite, then $\mu\left(F_{0}\right) \geq \mu\left(F_{q}\right)$ (and hence "=") for all except finitely many prime numbers $q \in \mathbb{Z}$.

The same holds for the Tjurina number.

Example 65. We illustrate the corollary by a simple example. Let $F=F_{0}=x^{p}+$ $x^{(p+1)}+y^{q}$ with $p, q$ prime numbers. Then $\mu\left(F_{0}\right)=(p-1)(q-1), \mu\left(F_{p}\right)=p(q-1)$ $\geq \mu\left(F_{0}\right)$ while $\mu\left(F_{q}\right)=\infty$. Moreover, for any prime number $r \neq p, q$ we have $\mu\left(F_{r}\right)=\mu\left(F_{0}\right)$.

\subsection{Determinacy of ideals}

Let $I$ be a proper ideal of $\mathbb{k}[[x]]$ and $f_{1}, \ldots, f_{m}$ a minimal set of generators of $I . I$ is called contact $k$-determined if for every ideal $J$ of $\mathbb{k}[[x]]$ that can be generated by $m$ elements $g_{1}, \ldots, g_{m}$ with $g_{i}-f_{i} \in\langle x\rangle^{k+1}$ for $i=1, \ldots, m$, the local $\mathbb{k}$-algebras $\mathbb{k}[[x]] / I$ and $\mathbb{k}[[x]] / J$ are isomorphic. $I$ is called finitely contact determined if $I$ is contact $k$-determined for some $k$. It is easy to see (cf. [GPh19, Proposition 4.3]) that these notions depend only on the ideal and not on the set of generators.

The ideal $I$ or the ring $\mathbb{k}[[x]] / I$ is called a complete intersection if $\operatorname{dim} \mathbb{k}[[x]] / I=$ $n-m$ and an isolated complete intersection singularity (ICIS) if it has moreover an isolated singularity.

Set $f=\left(f_{1}, \ldots, f_{m}\right) \in \mathbb{k}[[x]]^{m}$ and denote by $\left\langle\partial f / \partial x_{1}, \ldots, \partial f / \partial x_{n}\right\rangle$ the submodule of $\mathbb{k}[[x]]^{m}$, generated by the m-tuples $\partial f / \partial x_{i}=\left(\partial f_{1} / \partial x_{i}, \ldots, \partial f_{m} / \partial x_{i}\right)$, $i=1, \ldots, n$. We define

$$
T_{I}:=\mathbb{k}[[x]]^{m} / I \mathbb{k}[[x]]^{m}+\left\langle\partial f / \partial x_{1}, \ldots, \partial f / \partial x_{n}\right\rangle .
$$

If $I$ is a complete intersection, then $\tau(I):=\operatorname{dim}_{\mathbb{k}} T_{I}$ is called the Tjurina number of $I$. For a complete intersection $T_{I}$ is concentrated on the singular locus of 
$\mathbb{k}[[x]] / I$ (Definiton 59) and $\tau(I)$ is finite iff $I$ has an isolated singularity. This follows from [GPh19, Lemma 3.1], where it is shown that the ideals $I+I_{n-m}(\operatorname{Jac}(I))$ and $\operatorname{Ann}_{\mathbb{k}[[x]]}\left(T_{I}\right)$ have the same radical.

The module $T_{I}$ is used in the following theorem.

Theorem 66 ([GPh19], Theorem 4.6). Let $I \subset \mathbb{k}[[x]]$ be a proper ideal and $\mathbb{k}$ infinite. Then the following are equivalent:

(i) I is finitely contact determined.

(ii) $\operatorname{dim}_{\mathbb{k}} T_{I}<\infty$.

(iii) $R / I$ is an isolated complete intersection singularity.

If one of these condition is satisfied then $I$ is contact $\left(2 \operatorname{dim}_{\mathbb{k}} T_{I}-\operatorname{ord}(I)+2\right)$ determined, where ord $(I)=\max \left\{k \mid I \subset\langle x\rangle^{k}\right\}$. The implications (iii) $\Leftrightarrow($ ii $) \Rightarrow$ (i) hold for any field $\mathbb{k}$, as well as $(i) \Rightarrow$ (ii) for hypersurfaces.

Proposition 67. Let $A$ be Noetherian, $F_{1}, \ldots, F_{m} \in\langle x\rangle A[[x]]$. Let $I \subset A[[x]]$ be the ideal generated by $F_{1}, \ldots, F_{m}$ and $\hat{I}(\mathfrak{p}) \subset k(\mathfrak{p})[[x]], \mathfrak{p} \in \operatorname{Spec} A$, the ideal generated by $F_{1}(\mathfrak{p}), \ldots, F_{m}(\mathfrak{p}) \in k(\mathfrak{p})[[x]]$.

Assume that $V\left(I+I_{n-m}(\operatorname{Jac}(I))\right) \subset V(\langle x\rangle)$ (as sets), or $\operatorname{dim} A=1$, or $F_{i} \in\langle x\rangle A[x]$ for $i=1, \ldots, m$, or $A$ is a complete local ring containing a field.

Then any $\mathfrak{p} \in \operatorname{Spec} A$ has an open neighbourhood $U \subset \operatorname{Spec} A$ such that for all $\mathfrak{q} \in U \operatorname{dim}_{k(\mathfrak{p})} T_{\hat{I}(\mathfrak{p})} \geq \operatorname{dim}_{k(\mathfrak{q})} T_{\hat{I}(\mathfrak{q})}$.

Proof. By [GPh19, Lemma 3.1] $\operatorname{Supp}\left(T_{I}\right)=V\left(I+I_{n-m}(\operatorname{Jac}(I))\right)$. The claim follows from Corollary 45.

\subsection{Tjurina number of complete intersection singularities}

We show first that being a regular sequence in a flat family of power series in $R=A[[x]]$ is an open property.

Proposition 68. Let $A$ be a Noetherian ring, $F_{i} \in\langle x\rangle R, i=1, \ldots, m$ and $M a$ finitely generated $R$-module. For $\mathfrak{p} \in \operatorname{Spec} A$ we denote by $F_{i}(\mathfrak{p})$ the image of $F_{i}$

in $\hat{R}(\mathfrak{p})=k(\mathfrak{p})[[x]]$ and by $F_{\mathfrak{i n}_{\mathfrak{p}}}$ the image of $F_{i}$ in $R_{\mathfrak{n}_{\mathfrak{p}}}(\mathfrak{p})$ (cf. Definition 11).

(i) If $\mathfrak{p} \in \operatorname{Spec} A$ then $F_{1}(\mathfrak{p}), \ldots, F_{m}(\mathfrak{p})$ is an $\hat{M}(\mathfrak{p})$-sequence iff $F_{1 \mathfrak{n}_{\mathfrak{p}}}, \ldots, F_{m \mathfrak{n}_{\mathfrak{p}}}$ is an $M_{\mathfrak{n}_{\mathfrak{p}}}(\mathfrak{p})$-sequence.

(ii) Let $F_{1}, \ldots, F_{m}$ be an $M$-sequence and let $M /\left\langle F_{1}, \ldots, F_{m}\right\rangle M$ be A-flat. Then $F_{1}(\mathfrak{p}), \ldots, F_{m}(\mathfrak{p})$ is an $\hat{M}(\mathfrak{p})$-sequence for all $\mathfrak{p} \in \operatorname{Spec} A$.

(iii) Let $\mathfrak{p} \in \operatorname{Spec} A$ and $F_{1}(\mathfrak{p}), \ldots, F_{m}(\mathfrak{p})$ an $\hat{M}(\mathfrak{p})$-sequence. If $M /\left\langle F_{1}, \ldots, F_{m}\right\rangle M$ is flat over $A$, then there exists an open neighbourhood $U$ of $\mathfrak{p}$ in $\operatorname{Spec} A$ such that $F_{1}(\mathfrak{q}), \ldots, F_{m}(\mathfrak{q})$ is a $\hat{M}(\mathfrak{q})$-sequence for all $\mathfrak{q}$ in $U$. 
Proof. Set $M_{0}=M, M_{i}=M /\left\langle F_{1}, \ldots, F_{i}\right\rangle M$ and consider for $i=1, \ldots, m$ the exact sequence

$$
0 \rightarrow K_{i-1} \rightarrow M_{i-1} \stackrel{F_{i}}{\rightarrow} M_{i-1} \rightarrow M_{i} \rightarrow 0
$$

with $K_{i-1}$ the kernel of $F_{i}$. hence

(i) By Lemma $15.2 \hat{R}(\mathfrak{p})=R_{\mathfrak{n}_{\mathfrak{p}}}(\mathfrak{p})^{\wedge}$ and $\hat{M}_{i}(\mathfrak{p})=M_{i, \mathfrak{n}_{\mathfrak{p}}}(\mathfrak{p})^{\wedge}$ for all $i$ and

$$
F_{i}(\mathfrak{p})=F_{i \mathfrak{n}_{\mathfrak{p}}}^{\wedge}:\left(M_{i-1, \mathfrak{n}_{\mathfrak{p}}}(\mathfrak{p})\right)^{\wedge} \rightarrow\left(M_{i-1, \mathfrak{n}_{\mathfrak{p}}}(\mathfrak{p})\right)^{\wedge} .
$$

Since $M_{i, \mathfrak{n}_{\mathfrak{p}}}(\mathfrak{p})$ is a finite $R_{\mathfrak{n}_{\mathfrak{p}}}$-module we have (by [AM69, Theorem 10.13]) $\hat{M}_{i}(\mathfrak{p})=$ $M_{i, \mathfrak{n}_{\mathfrak{p}}}(\mathfrak{p}) \otimes_{R_{\mathfrak{n}_{\mathfrak{p}}}} R_{\mathfrak{n}_{\mathfrak{p}}}^{\wedge}$. Moreover $R_{\mathfrak{n}_{\mathfrak{p}}}^{\wedge}$ is faithfully flat over the local ring $R_{\mathfrak{n}_{\mathfrak{p}}}$ ([Mat86, Theorem 8.14]). Hence $F_{i \mathfrak{n}_{\mathfrak{p}}}: M_{i-1, \mathfrak{n}_{\mathfrak{p}}}(\mathfrak{p}) \rightarrow M_{i-1, \mathfrak{n}_{\mathfrak{p}}}(\mathfrak{p})$ is injective iff $F_{i}(\mathfrak{p})$ : $\hat{M}_{i-1}(\mathfrak{p}) \rightarrow \hat{M}_{i-1}(\mathfrak{p})$ is injective.

(ii) By assumption $K_{i-1}=0$ for $i=1, \ldots, m$ and $M /\left\langle F_{1}, \ldots, F_{m}\right\rangle M$ is $A$ flat. By Lemma 16 the Jacobson radical of $R$ contains $\langle x\rangle$ and $F_{1}, \ldots, F_{m}$ is a regular sequence contained in the Jacobson radical. Hence $M_{i}$ is $A$-flat and $M_{i, \mathfrak{n}_{\mathfrak{p}}}$ is $A_{\mathfrak{p}}$-flat for all $i$ (repeated application of [Mat86, Theorem 22.2]). Tensoring $0 \rightarrow M_{i-1, \mathfrak{n}_{\mathfrak{p}}} \rightarrow M_{i-1, \mathfrak{n}_{\mathfrak{p}}} \rightarrow M_{i, \mathfrak{n}_{\mathfrak{p}}} \rightarrow 0$ with $\otimes_{A_{\mathfrak{p}}} k(\mathfrak{p})$ we get an exact sequence $\left.0 \rightarrow M_{i-1, \mathfrak{n}_{\mathfrak{p}}}(\mathfrak{p}) \rightarrow M_{i-1, \mathfrak{n}_{\mathfrak{p}}}(\mathfrak{p})\right) \rightarrow M_{i, \mathfrak{n}_{\mathfrak{p}}}(\mathfrak{p}) \rightarrow 0$ for $i=1, \ldots m$ by [Mat86, Theorem 22.3]. Now apply (i).

(iii) Localizing the exact sequence $(*)$ at $\mathfrak{n}_{\mathfrak{p}}$ we get an exact sequence of finite $R_{\mathfrak{n}_{\mathfrak{p}}}$-modules. Taking the $\langle x\rangle$-adic completion, the sequence stays exact and we see that $\left(K_{i-1, \mathfrak{n}_{\mathfrak{p}}}\right)^{\wedge}=\operatorname{ker}\left(F_{i \mathfrak{n}_{\mathfrak{p}}}^{\wedge}:\left(M_{i-1, \mathfrak{n}_{\mathfrak{p}}}\right)^{\wedge} \rightarrow\left(M_{i-1, \mathfrak{n}_{\mathfrak{p}}}\right)^{\wedge}\right)$. By Lemma 15 $\hat{M}_{i-1}(\mathfrak{p})=\left(M_{i-1, \mathfrak{n}_{\mathfrak{p}}}\right)^{\wedge} \otimes_{A_{\mathfrak{p}}} k(\mathfrak{p})$ and $F_{i}(\mathfrak{p})=F_{i \mathfrak{n}_{\mathfrak{p}}}^{\wedge} \otimes_{A_{\mathfrak{p}}} k(\mathfrak{p})$, and by assumption $F_{i}(\mathfrak{p})$ is injective. We apply now repeatedly [Mat86, Theorem 22.5] to $A_{\mathfrak{p}} \rightarrow$ $\hat{R}_{\mathfrak{n}_{\mathfrak{p}}}=A_{\mathfrak{p}}[[x]]$ and to $F_{i \mathfrak{n}_{\mathfrak{p}}}^{\wedge}$ to get that $\left(K_{i-1, \mathfrak{n}_{\mathfrak{p}}}\right)^{\wedge}=K_{i-1, \mathfrak{n}_{\mathfrak{p}}} \otimes_{R_{\mathfrak{n} \mathfrak{p}}} R_{\mathfrak{n}_{\mathfrak{p}}}^{\wedge}=0$ and that $\left(M_{i, \mathfrak{n}_{\mathfrak{p}}}\right)^{\wedge}=M_{i, \mathfrak{n}_{\mathfrak{p}}} \otimes_{R_{\mathfrak{n}_{\mathfrak{p}}}} R_{\mathfrak{n}_{\mathfrak{p}}}^{\wedge}$ is flat over $A_{\mathfrak{p}}$ for all $i$. Since $R_{\mathfrak{n}_{\mathfrak{p}}}^{\wedge}$ is faithfully flat over $R_{\mathfrak{n}_{\mathfrak{p}}}$ this implies $K_{i-1, \mathfrak{n}_{\mathfrak{p}}}=0$ and that $M_{i, \mathfrak{n}_{\mathfrak{p}}}$ is flat over $A_{\mathfrak{p}}$.

The support of the $R$-module $K_{i-1}$ is closed and hence $\left(K_{i-1}\right)_{\mathfrak{n}_{\mathfrak{q}}}^{\wedge}=0$ for $\mathfrak{q}$ in an open neighbourhood $U$ of $\mathfrak{p}$ in Spec $A$. Moreover the flatness of $M /\left\langle F_{1}, \ldots, F_{m}\right\rangle M$ implies that $M_{\mathfrak{n}_{\mathfrak{q}}}^{\wedge} /\left\langle F_{1}, \ldots, F_{m}\right\rangle M_{\mathfrak{n}_{\mathfrak{q}}}^{\wedge}$ is $A_{\mathfrak{q}}$-flat. Applying [Mat86, Theorem 22.5] now to $F_{i \mathfrak{n}_{\mathfrak{q}}}^{\wedge}:\left(M_{i-1}\right)_{\mathfrak{n}_{\mathfrak{q}}}^{\wedge} \rightarrow\left(M_{i-1}\right)_{\mathfrak{n}_{\mathfrak{q}}}^{\wedge}$ we get that $\hat{M}_{i-1}(\mathfrak{q}) \rightarrow \hat{M}_{i-1}(\mathfrak{q})$ is injective and that $F_{1}(\mathfrak{q}), \ldots, F_{m}(\mathfrak{q})$ is an $\hat{M}(\mathfrak{q})$-sequence.

Proposition 69. Let $A$ be a Noetherian ring and $I \subset\langle x\rangle A[[x]]$ an ideal generated by $F_{1}, \ldots, F_{m}$, such that $A[[x]] / I A[[x]]$ is A-flat. For $\mathfrak{p} \in \operatorname{Spec} A$ denote by $\hat{I}(\mathfrak{p}) \subset$ $k(\mathfrak{p})[[x]]$ the ideal generated by $F_{1}(\mathfrak{p}), \ldots, F_{m}(\mathfrak{p})$.

1. If $\hat{I}(\mathfrak{p})$ is a complete intersection, then $\hat{I}(\mathfrak{q})$ is a complete intersection for $\mathfrak{q}$ in an open neighbourhood of $\mathfrak{p}$ in $\operatorname{Spec} A$.

2. Assume that $\hat{I}(\mathfrak{p})$ is an ICIS and that the hypotheses of Proposition 67 are satisfied. Then $\hat{I}(\mathfrak{q})$ is an ICIS with $\tau(I(\mathfrak{p})) \geq \tau(I(\mathfrak{q}))$ for $\mathfrak{q}$ in an open neighbourhood of $\mathfrak{p}$ in $\operatorname{Spec} A$. 
Proof. 1. We may assume that $F_{1}(\mathfrak{p}), \ldots, F_{m}(\mathfrak{p})$ is a $k(\mathfrak{p})[[x]]$-sequence. By Proposition $68 F_{1}(\mathfrak{q}), \ldots, F_{m}(\mathfrak{q})$ is a $k(\mathfrak{q})[[x]]$-sequence, hence $\hat{I}(\mathfrak{q})$ is a complete intersection, for $\mathfrak{q}$ in an open neighbourhood of $\mathfrak{p}$ in $\operatorname{Spec} A$.

2. follows from Proposition 67 since for $\hat{I}(\mathfrak{q}) \subset k(\mathfrak{q})[[x]]$ a complete intersection $\operatorname{dim}_{k(\mathfrak{q})} T_{\hat{I}(\mathfrak{q})}$ is the Tjurina number of $\hat{I}(\mathfrak{q})$.

\section{References}

[AM69] Atiyah, M.F., Macdonald I.G.: Introduction to commutative algebra. AddisonWesley, 1969.

[BGM11] Boubakri, Y.; Greuel, G.-M.; Markwig, T. Normal Forms of Hypersurface Singularities in Positive Characteristic. Mosc. Math. J. 11, No. 4, 657-683 (2011).

[BGM12] Boubakri, Y.; Greuel, G.-M.; Markwig, T. Invariants of hypersurface singularities in positive characteristic. Rev. Mat. Complut. 25, No. 1, 61-85 (2012).

[DGPS] Decker, W.; Greuel, G.-M.; Pfister, G.; Schönemann, H.: SingulaR 4-1-2 - A computer algebra system for polynomial computations. http://www.singular.uni-kl.de (2019).

[Eis95] Eisenbud, D.; Commutative algebra. With a view toward algebraic geometry. Graduate Texts in Mathematics 150. Springer (1995).

[GR71] Grauert, H..; Remmert, R.: Analytische Stellenalgebren, Grundlehren der mathematischen Wissenschaften, Springer (1971).

[GLS07] Greuel, G.-M.; Lossen, C.; Shustin, E.: Introduction to Singularities and Deformations. Springer (2007).

[GP08] Greuel, G.-M., Pfister, G.: A Singular Introduction to Commutative Algebra, 2nd Edition, Springer, Berlin (2008). With contributions by O. Bachmann, C. Lossen and H. Schönemann.

[GPh19] Greuel, G.-M.; Pham, T.H.: Finite determinacy of matrices and ideals in arbitrary characteristics, J. Algebra 530 (2019).

[KPR75] Kurke, H.; Pfister, G.; Roczen, M.: Henselsche Ringe und algebraische Geometrie. Mathematische Monographien. Band 11. Berlin: VEB Deutscher Verlag der Wissenschaften. 223 S. M 57.00 (1975).

[KPP78] Kurke, H.; Pfister, G.; Popescu, D; Roczen, M.; Mostowski T.: Die Approximationseigenschaft lokaler Ringe. Lecture Notes in Mathematics 634, SpringerVerlag Berlin Heidelberg New York (1978).

[La67] Lafou, J. P. : Anneaux henseliens et théorèms de préparation. C. R. Acad. Sci., Paris, A1161 -A1162 (1967).

[Maj10] Majadas, J., Rodicio, A.G.: : Smoothness, Regularity and Complete Intersection. LN London Math. Soc. 373 (2010).

[Mat86] Matsumura, H.: Commutative ring theory. Cambridge Univ. Press (1986).

[Pf07] Pfister, G. On modular computation of standard basis. An. Stiint. Univ. Ovidius Constana, Ser. Mat. 15, No. 1, 129-138 (2007).

[Sa71] Saito, K. Quasihomogene isolierte Singularitäten von Hyperflächen. Invent. Math. 14, 123-142 (1971). 
[Sta19] The Stacks project authors. The Stacks project (2019) https://stacks.math.columbia.edu

Gert-Martin Greuel and Gerhard Pfister

University of Kaiserslautern

Department of Mathematics

Erwin-Schroedinger Str.

67663 Kaiserslautern

Germany

e-mail: greuel@mathematik.uni-kl.de

e-mail: pfister@mathematik.uni-kl.de 